\title{
Mineral chemistry and crystallization parameters of the A-type Paleoproterozoic Bannach Granite, Carajás Province, Pará, Brazil
}

\author{
Caio José Soares Mesquita ${ }^{1 *}$, Roberto Dall'Agnol ${ }^{1,2}$, \\ José de Arimatéia Costa de Almeida ${ }^{3}$
}

\begin{abstract}
The A-type Paleoproterozoic Bannach Granite belongs to the Jamon Suite and cross-cut the Archean units of the Rio Maria Domain in the Carajás Province. It comprises eight facies with varied mafic content and texture: even-grained cumulate granite (CG), even coarse-grained biotite-amphibole monzogranite (cBAMz), even coarse-grained amphibole-biotite monzogranite (cABMz), porphyritic biotite monzogranite ( $p B M z)$, even coarse-grained leucomonzogranite ( $(L M z)$, early even medium-grained leucomonzogranite (EmLMz), late even medium-grained leucomonzogranite $(L m L M z)$ and even fine-grained leucomonzogranite ( $f L M z)$. In the less evolved facies, the dominant amphibole is Fe-hornblende passing to Fe-edenite or hastingsite, with associated cummingtonitelgrunerite originated from the destabilization of clinopyroxene. Biotite has ferroan composition and approaches annite in the late-emplaced leucomonzogranite. Plagioclase varies from andesine to oligoclase or from oligoclase to albite. Titanite, magnetite, and ilmenite are found in all granite facies showing magmatic origin. The near liquidus temperatures vary between $943^{\circ} \mathrm{C}$ and $795^{\circ} \mathrm{C}$ and the estimated emplacement pressure is $300 \pm 50 \mathrm{MPa}(-11.1 \pm 1.9 \mathrm{~km})$. Amphibole $\mathrm{Fe} /(\mathrm{Fe}+\mathrm{Mg})$ ratios together with magmatic magnetite and titanite indicate that these granites crystallized at moderately oxidizing conditions, similar to those admitted for the Jamon granite and magnetite series granites of Laurentia. However, the composition of biotite suggests a more reduced character close to NNO to NNO -0.5. The late leucomonzogranite facies is an exception because it displays high $\mathrm{Fe} /(\mathrm{Fe}+\mathrm{Mg})$ ratios in biotite and approaches to the reduced granites of Carajás, Laurentia and Fennoscandia in this regard. It derived from a more reduced source than the other facies of Bannach Granite.
\end{abstract}

KEYWORDS: mineral chemistry; crystallization parameters; A-type granite; Bannach; Paleoproterozoic; Carajás Province.

\section{INTRODUCTION}

At the end of Paleoproterozoic and during the Mesoproterozoic Era, an intense magmatic activity has been reported in several cratonic areas of the world. These magmatic events are remarkable in the Amazonian Craton, where they include a huge volume of granitic and subordinate mafic plutonic rocks, associated with felsic, intermediate, and mafic volcanic rocks (Dall'Agnol et al. 1999a, and references therein). In the Carajas Province, the magmatism is Paleoproterozoic in age and has anorogenic character. It is represented by the Jamon, Velho Guilherme, and Serra dos Carajás suites (Dall'Agnol et al. 2005) and the Seringa (Paiva
Jr. et al. 2011), São João (Lima et al. 2014) and Gradaús (Carvalho 2017) granites.

The objective of this work was to study the mineralogy of the main facies of Bannach Granite (Duarte 1992, Almeida et al. 2006, 2007), a batholith hosted by Archaean granitoid rocks of Rio Maria Domain, southeast of Amazonian Craton. The pluton belongs to Jamon Suite, which is composed of oxidized A-type granites (Dall'Agnol et al. 2005, Dall'Agnol \& Oliveira 2007). Petrographic, geochemical and magnetic petrology studies (Almeida et al. 2006, 2007) allowed to determine the spatial distribution and geochemical characteristics of the facies in the pluton, as well as to estimate processes of magmatic differentiation. In addition, geophysical data indicated that

${ }^{1}$ Programa de Pós-Graduação em Geologia e Geoquímica, Instituto de Geociências, Universidade Federal do Pará - Belém (PA), Brazil. E-mail: caiojsm@gmail.com, robdal@ufpa.br

${ }^{2}$ Instituto Tecnológico Vale - Belém (PA), Brazil.

${ }^{3}$ Instituto de Geociências e Engenharias, Universidade Federal do Sul e Sudeste do Pará - Marabá (PA), Brazil. E-mail: ari@unifesspa.edu.br

*Corresponding author.

Manuscript ID: 20170082 Received on: 06/21/2017. Approved on: 03/12/2018. 
the pluton is a sheeted, laccolithic body (Oliveira et al. 2008). However, the mineral chemistry investigation in Jamon Suite is restricted to Jamon Granite (Dall'Agnol et al.1999c), and similar studies in the other plutons are still lacking.

Mineral chemistry is an important tool to estimate crystallization parameters of granitic magmas. The Al-in-hornblende geobarometer was proposed in the 1980s (Hammarstron \& Zen 1986, Hollister et al. 1987, Johnson \& Rutherford 1989, Schmidt 1992) and was applied to several granitoid rocks (Anderson \& Smith 1995; Lamarão \& Dall'Agnol 2004, Anderson \& Morrison 2005, Anderson et al. 2008). Zircon saturation (Watson \& Harrison 1983, Miller et al. 2003) and amphibole-plagioclase (Blundy \& Holland 1990) geothermometers have also been used to determine crystallization temperatures in many igneous rocks. Recent works are estimating or discussing crystallization parameters based on the chemical composition of the main mineral phases in granitoids rocks (Ridolfi et al. 2010, Ridolfi \& Rezulli 2012, Erdmann et al. 2014, Cunha et al. 2016, Putirka 2016, Mutch et al. 2016, for specific evaluations of the Al-in-hornblende geobarometer). The oxygen fugacity that dominates during the magmatic evolution can be estimated using $\mathrm{Fe} /(\mathrm{Fe}+\mathrm{Mg})$ ratios in amphibole and biotite, given this ratio is strongly dependent of oxygen fugacity (Anderson \& Smith 1995, Dall'Agnol et al. 1999b, 1999c, 2005, 2017, Anderson \& Morrison 2005, Dall'Agnol \& Oliveira 2007, Anderson et al. 2008, Cunha et al. 2016). The composition of primary magnetite-ilmenite pairs and the nature of Fe-Ti oxide minerals formed during the magmatic stage are also useful to establish constraints about the oxygen fugacity prevalent in crystallization (Ishihara 1981, Wones 1989, Frost \& Lindsley 1991, Frost 1991, Clark 1999, Dall'Agnol et al. 1997, 1999b, 2005 , Dall'Agnol \& Oliveira 2007).

Thus, our approach has been to determine the compositions of the main minerals phases in the dominant facies of Bannach Granite, in order to estimate crystallization parameters prevalent during the petrological evolution of this pluton. In addition, we compare the obtained data with similar granites of the Carajás Province and of other cratons. Therefore, this work intends to contribute to a better mineralogical characterization of the Paleoproterozoic magmatism of Carajás Province and to clarify the petrological evolution of the oxidized A-type granites in general.

\section{GEOLOGICAL SETTING}

The Carajás Province (CP) is the most important Archean terrain of Amazonian Craton (Fig. 1A). It was initially subdivided into Rio Maria Domain (RMD), in the south, and Carajás Domain (CD), in the north (Vasquez et al. 2008).
However, CD was recently divided into three different domains (Dall'Agnol et al. 2013):

- Sapucaia (2.95 to $2.73 \mathrm{Ga}$ );

- Canaã dos Carajás (3.0 to $2.73 \mathrm{Ga}$ );

- Carajás Basin (2.75 to $2.73 \mathrm{Ga}$ ).

Rio Maria Domain (3.0 to 2.86 Ga; Macambira \& Lafon 1995; Fig. 1B) is composed of greenstone belts (Andorinhas Supergroup; Huhn et al. 1988), and four groups of Archean granitoids:

- Tonalitic-trondhjemitic series (TTG) dated between 2.98 and $2.91 \mathrm{Ga}$, possibly in two different episodes (Almeida et al. 2011);

- High-Mg granodiorites and associated rocks (sanukitoids; ca 2.87 Ga; Althoff et al. 2000, Leite 2001, Oliveira et al. 2009b);

- High-Ba and Sr calc-alkaline leucogranodiorites and leucomonzogranites (ca $2.87 \mathrm{Ga}$; Almeida et al. 2010);

- Potassic leucogranites (ca 2.86 Ga; Almeida et al. 2013).

In RMD (Fig. 1B), Archean units are cut by the Paleoproterozoic anorogenic granites of Jamon Suite (Dall'Agnol et al. 2005). This suite is represented by six isotropic granites, Marajoara (Rocha Jr. 2004), Jamon (Dall'Agnol et al. 1997, 1999b, 1999c), Musa (Gastal 1987, Dall'Agnol et al. 1999b), Manda Saia (Leite 2001), Bannach (Huhn et al. 1988, Duarte 1992, Almeida et al. 2006, 2007) and Redenção (Montalvão et al. 1982, Barbosa et al. 1995, Oliveira et al. 2009a, 2010a). The granitic plutons have discordant contacts with the country rocks and were emplaced at high crustal level. These plutons are composed of A-type oxidized (Dall'Agnol et al. 1999b, 1999c, 2005, Dall'Agnol \& Oliveira 2007) monzogranites with subordinated syenogranites (Gastal 1987, Dall'Agnol et al. 1999b, 2005, Almeida et al. 2006, 2007, Oliveira et al. 2009a).

The Archean country rocks were affected by contact metamorphism in the facies hornblende hornfels (Dall'Agnol et al. 2005). U-Pb (TIMS), Pb-evaporation and $\mathrm{Pb}-\mathrm{Pb}$ (whole-rock) data obtained for the Jamon Suite granites indicated that they crystallized around $1.88 \mathrm{Ga}$ (Machado et al. 1991, Barbosa et al. 1995, Dall'Agnol et al. 1999a). Coeval mafic, intermediate and felsic dikes are associated with Jamon Suite (Gastal 1987, Dall'Agnol et al. 2005).

\section{GEOLOGY AND PETROGRAPHY OF THE BANNACH GRANITE}

\section{Geology}

The Bannach batholith has an ellipsoidal shape in plant with a main axis measuring approximately $40 \mathrm{~km}$ along a NNW trend (Fig. 1C), cutting the E-W structural trend of 
the host Archean rocks. Almeida et al. (2006) and Oliveira et al. (2008) suggest that the Bannach batholith is a composite intrusion formed by three coalescent plutons oriented in the NW-SE direction.

Based on modal content of mafic minerals and texture, eight monzogranitic facies have been described in Bannach Granite (Almeida et al. 2006). The less evolved granites facies tend to occupy the outer zones and the more evolved ones, the central area of the body (Fig. 1C). These facies are: even-grained cumulate granite (CG), even coarse-grained biotite-amphibole monzogranite (cBAMz), even coarsegrained amphibole-biotite monzogranite (cABMz), porphyritic biotite monzogranite (pBMz), even coarse-grained leucomonzogranite (cLMz), early even medium-grained leucomonzogranite (EmLMz), late even medium-grained leucomonzogranite (LmLMz) and even fine-grained leucomonzogranite (fLMz). Petrographic features from a previous study (Almeida et al. 2006) are integrated with complementary observations in this work.

The CG are coarse-grained rocks occurring as enclaves with sharp contacts in the cBAMz. The latter is commonly associated with the $\mathrm{CABMz}$ and occurs as enclaves in the pBMz and fLMz facies. The cBAMz, cABMz and cLMz facies display a gradation from the more mafic to more felsic facies, that suggests evolution by fractional crystallization process. Rocks of the pBMz facies have locally rapakivi texture near

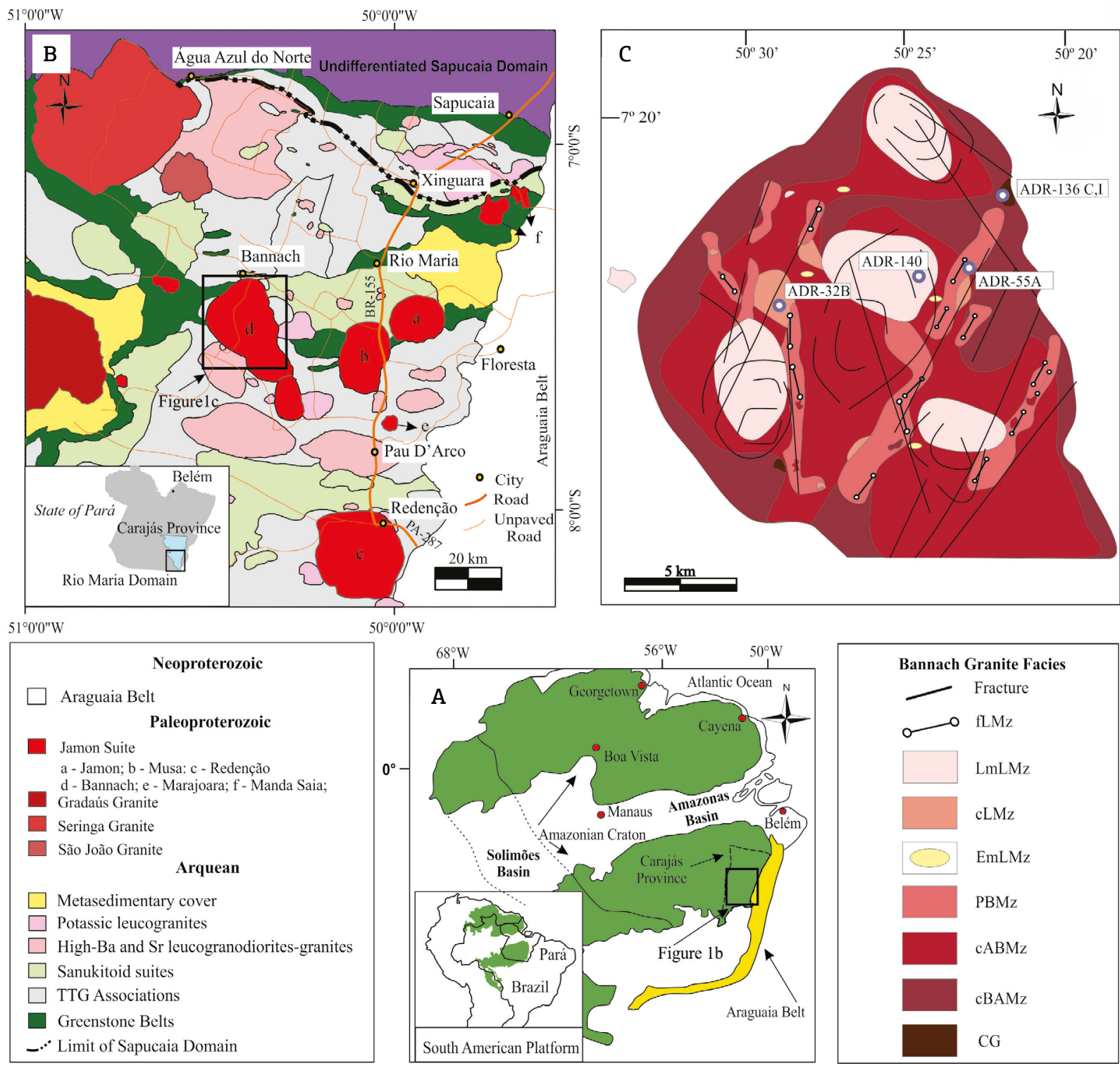

Figure 1. (A) Location of the Carajás Province in the Amazonian Craton; (B) geological map of the Rio Maria Domain modified from Dall'Agnol et al. 2006; M.A. Oliveira et al. 2009b; Almeida et al. 2011; (C) geological map showing the areal distribution of the main facies in the northern part of Bannach pluton (Almeida et al. 2006), with the location of samples selected for microprobe analyzes. 
gradational contacts with cLMz and around oval enclaves of $\mathrm{cLMz}$ which indicates mingling between these facies.

The fLMz rocks often occur as veins cross-cutting the cBAMz, cLMz, and pBMz, but transitional contacts between the fLMz and $\mathrm{pBMz}$ facies were also observed. The medium leucomonzogranites were divided into two facies. On the one hand, an earlier facies (EmLMz) occurs as enclaves in the $\mathrm{CABMz}$ without mingling evidence. On the other hand, the leucomonzogranites outcropping in oval structures in the central part of the pluton (Fig. 1C) were believed to be late in the crystallization (LmLMz) of Bannach Granite.

Geophysical studies demonstrated that the Bannach Granite is tabular in shape and evolved through three magmatic pulses (Oliveira et al., 2008). In the first stage, the magma ascent took place by dikes and originated the $\mathrm{cBAMz}$ and $\mathrm{CABMz}$ facies. In the second stage, the ascent of a younger and evolved magma happened, generating the $\mathrm{pBMz}$ and fLMz. In the third stage, annular faults served as channels for the migration and emplacement of the LmLMz.

\section{Petrography}

A detailed petrographic study of polished thin sections of 17 representative samples of the main facies exposed in the northern part of Bannach batholith was done. Optical microscopy was performed using transmitted and reflected light. Modal analyses (2,000 points on average per thin section) were performed by the authors (Tab. 1), but one available composition of the LmLMz was also employed (Almeida et al. 2006). The rocks were classified according to the recommendations of the Subcommission on the Systematics of Igneous Rocks of the IUGS (Le Maitre et al. 2002).

The QAP and Q - (A + P) - M' diagrams (Le Maitre et al. 2002) show that all the analyzed rocks can be classified as monzogranites (Fig. 2). Except for the CG facies, all petrographic facies of Bannach are hololeucocratic to leucocratic and constituted by similar proportions of quartz, plagioclase and $\mathrm{K}$-feldspar. Biotite is the dominant mafic mineral, found in all granite varieties; amphibole is abundant only in the CG and cBAMz facies and is scarce or absent in the leucomonzogranite facies. Zircon, titanite, apatite, allanite, ilmenite, and magnetite are the primary accessory phases. Chlorite, white micas, epidote, and fluorite occur as secondary phases.

In the Bannach Granite (Almeida et al. 2006), the decrease of modal mafic mineral content from the less evolved facies to the leucogranites is followed by moderate increase of quartz content and simultaneous decrease of both, plagioclase/potassium feldspar ratio and anorthite content of plagioclase. This suggests that the Bannach Granite magmatic differentiation was controlled by fractional crystallization.
Due to the compositional and textural similarities between some Bannach facies, we are going to describe these rocks in three groups (see detailed description in Almeida et al. 2006).

\section{Biotite-amphibole-bearing facies (CG, cBAMz, cABMz, cLMz)}

These rocks have equigranular coarse-grained hypidiomorphic texture with locally porphyritic texture. The K-feldspar modal content increases while mafic minerals contents decreases following the trend: CG-cBAMz-cABMz-cLMz (Tab. 1). Aggregates of mafic minerals and plagioclase are common in these rocks, especially in the CG and cBAMz. Crystals of calcic amphibole include fine-grained ferromagnesian amphibole derived from clinopyroxene (Fig. 3A). These features are rare or absent in $\mathrm{cABMz}$ and $\mathrm{cLMz}$ where biotite is the dominant mafic mineral.

According to Almeida et al. (2006), plagioclase compositions in this group range from sodic andesine $\left(\mathrm{An}_{30}\right)$ to calcic oligoclase $\left(\mathrm{An}_{24}\right)$ in the crystal core and sodic oligoclase $\left(\mathrm{An}_{15}\right)$

to pure albite $\left(\mathrm{An}_{0}\right)$ at the rim, showing a normal zoning. The evidences of a more evolved character of the cLMz are: amphibole occurs only as fine-grained relic crystals; chloritization of biotite is relatively more intense; granophyric textures are common; plagioclase crystals are more sodic $($ An $\leq 20)$.

\section{Porphyritic biotite monzogranite (pBMz)}

This facies consists of euhedral to subeuhedral phenocrysts of quartz, plagioclase, and K-feldspar with dimensions ranging from 2 to $20 \mathrm{~mm}$ immersed in a quartz-feldspar matrix (50 to $80 \%$ of the rock) with subordinate mafic mineral (Fig. 3B). In the matrix, granophyric textures are common (Fig. 3C). Occasionally, plagioclase phenocrysts show growth zones and evidence of normal zoning with altered calcic core and preserved sodic rim (Fig. 3D). Almeida et al. (2006) observed that the composition of the plagioclase of the matrix is similar to that of the borders of plagioclase crystals of the biotite-amphibole bearing facies (sodic oligoclase to albite; $\mathrm{An}_{16-5}$ ). Embayed quartz phenocrysts frequently occur and indicate changes in the magmatic conditions causing destabilization of quartz, followed by a new crystallization stage in the matrix (Fig. 3E). Mafic and plagioclase aggregates are noted, although they are scarce. Biotite chloritization is remarkable.

\section{Medium- to fine-grained leucomonzogranites (EmLMz, LmLMz, fLMz)}

Both EmLMz and LmLMz facies display equigranular medium-grained texture and are hololeucocratic. However, the 
Caio José Soares Mesquita et al.

Table 1. Modal compositions** of different facies of the Bannach Granite.

\begin{tabular}{|c|c|c|c|c|c|c|c|c|c|}
\hline \multirow{2}{*}{ Facies Minerals } & \multirow{2}{*}{$\begin{array}{c}\text { CG } \\
\text { ADR-136i }\end{array}$} & \multicolumn{3}{|c|}{ cBAMz } & \multicolumn{2}{|c|}{ cABMz } & \multicolumn{3}{|c|}{ PBMz } \\
\hline & & ADR-136C & ADR-26 & ADR-67 & ADR-55A & ADR-100A & ADR-35B & ADR-35B1 & ADR-19 \\
\hline Quartz & 26,30 & 29,70 & 30,15 & 28,30 & 35,72 & 38,70 & 41,05 & 40,65 & 39,40 \\
\hline K-feldspar & 13,05 & 23,05 & 22,30 & 27,99 & 25,94 & 19,90 & 32,30 & 30,70 & 34,70 \\
\hline Plagioclase & 20,65 & 33,60 & 31,20 & 32,57 & 32,44 & 32,20 & 24,25 & 24,70 & 21,55 \\
\hline Biotite & 12,90 & 3,75 & 4,60 & 2,30 & 3,61 & 5,40 & 0,45 & 0,30 & 2,10 \\
\hline Hornblende & 16,95 & 6,90 & 9,50 & 7,07 & 0,94 & 1,50 & - & - & 1,40 \\
\hline Fe-Mg Amphibole & 2,20 & 0,20 & 0,25 & 0,21 & $\mathrm{x}$ & 0,15 & - & - & $\mathrm{x}$ \\
\hline Opaque minerals & 6,45 & 2,30 & 1,40 & 1,00 & 0,95 & 0,75 & 0,05 & 0,40 & 0,20 \\
\hline Titanite & - & - & 0,15 & 0,14 & 0,06 & 0,05 & - & - & 0,05 \\
\hline Allanite & 0,25 & 0,10 & 0,10 & 0,07 & 0,11 & 0,15 & - & 0,05 & 0,10 \\
\hline Chlorite & - & 0,05 & - & - & - & 0,85 & 1,45 & 2,40 & 0,10 \\
\hline Muscovite & - & $\mathrm{x}$ & $\mathrm{x}$ & - & 0,06 & - & $\mathrm{x}$ & 0,05 & $\mathrm{x}$ \\
\hline Fluorite & 0,65 & 0,05 & 0,10 & 0,07 & 0,06 & 0,20 & 0,05 & 0,40 & 0,05 \\
\hline Intergrains albite & 0,10 & 0,10 & $\mathrm{x}$ & 0,14 & $\mathrm{x}$ & 0,05 & 0,40 & 0,30 & 0,30 \\
\hline Acessory (Ap + Zrn) & 0,50 & 0,20 & 0,25 & 0,14 & 0,11 & 0,10 & $x$ & 0,05 & 0,05 \\
\hline Felsic & 60,10 & 86,45 & 83,65 & 89,00 & 94,10 & 90,85 & 98,00 & 96,35 & 95,95 \\
\hline Mafic & 39,90 & 13,55 & 16,35 & 11,00 & 5,90 & 9,15 & 2,00 & 3,65 & 4,05 \\
\hline \multicolumn{10}{|l|}{ To $100 \%$} \\
\hline Quartz & 43,83 & 34,40 & 36,04 & 31,85 & 37,96 & 42,62 & 42,06 & 42,32 & 41,19 \\
\hline K-feldspar & 21,75 & 26,69 & 26,66 & 31,50 & 27,57 & 21,92 & 33,09 & 31,96 & 36,28 \\
\hline Plagioclase & 34,42 & 38,91 & 37,30 & 36,65 & 34,47 & 35,46 & 24,85 & 25,72 & 22,53 \\
\hline \multirow{2}{*}{ Minerals } & \multicolumn{3}{|c|}{ cLMz } & LmLMz & \multicolumn{3}{|c|}{ EmLMz } & \multicolumn{2}{|c|}{ fLMz } \\
\hline & ADR-28 & ADR-32B & ADR-32B1 & ADR-27C* & ADR-140 & ADR-140-1 & ADR-52 & ADR-37 & ADR-35A \\
\hline Quartz & 35,90 & 29,50 & 21,00 & 32,10 & 37,00 & 36,35 & 32,40 & 39,35 & 39,80 \\
\hline K-feldspar & 32,90 & 40,05 & 43,70 & 42,00 & 32,70 & 33,70 & 36,05 & 30,70 & 32,30 \\
\hline Plagioclase & 26,70 & 26,95 & 31,70 & 23,50 & 25,15 & 25,45 & 29,20 & 28,40 & 25,55 \\
\hline Biotite & 2,95 & 1,75 & 1,60 & 1,20 & 3,25 & 2,50 & 1,40 & 0,70 & 0,65 \\
\hline Hornblende & 0,75 & 0,05 & 0,60 & 0,50 & - & - & - & - & - \\
\hline Fe-Mg Amphibole & $\mathrm{x}$ & - & 0,05 & - & - & - & - & - & - \\
\hline Opaque minerals & 0,30 & 0,45 & 0,35 & 0,20 & 0,35 & 0,40 & 0,20 & $\mathrm{x}$ & 0,10 \\
\hline Titanite & 0,05 & - & 0,05 & - & - & - & - & - & - \\
\hline Allanite & 0,10 & - & 0,10 & - & 0,15 & 0,30 & - & - & - \\
\hline Chlorite & - & 0,75 & 0,15 & 1,50 & 0,15 & 0,25 & 0,60 & 0,65 & 1,05 \\
\hline Muscovite & $\mathrm{x}$ & 0,05 & - & - & 0,15 & 0,15 & - & 0,20 & - \\
\hline Fluorite & 0,05 & - & 0,25 & _- & 0,60 & 0,30 & _- & - & 0,55 \\
\hline Intergrains albite & 0,15 & 0,40 & 0,35 & 0,50 & 0,20 & 0,50 & 0,10 & - & - \\
\hline Acessory (Ap + Zrn) & 0,15 & 0,05 & 0,10 & - & 0,30 & 0,10 & 0,05 & - & - \\
\hline Felsic & 95,65 & 96,90 & 96,75 & 98,10 & 95,05 & 96,00 & 97,75 & 98,45 & 97,65 \\
\hline Mafic & 4,35 & 3,10 & 3,25 & 3,40 & 4,95 & 4,00 & 2,25 & 1,55 & 2,35 \\
\hline \multicolumn{10}{|l|}{ To $100 \%$} \\
\hline Quartz & 37,59 & 30,57 & 21,78 & 32,89 & 39,01 & 38,06 & 33,18 & 39,97 & 40,76 \\
\hline K-feldspar & 34,45 & 41,50 & 45,33 & 43,03 & 34,48 & 35,29 & 36,92 & 31,18 & 33,08 \\
\hline Plagioclase & 27,96 & 27,93 & 32,88 & 24,08 & 26,52 & 26,65 & 29,90 & 28,85 & 26,16 \\
\hline
\end{tabular}

CG: even-grained cumulatic granite; cBAMz: even coarse-grained biotite-amphibole monzogranite; cABMz: even coarse-grained amphibolebiotite monzogranite; PBMz: porphyritic biotite mongranite; cLMz: even coarse-grained leucomonzogranite; EmLMz: early even medium-grained leucomonzogranite; LmLMz: late even medium-grained leucomonzogranite; fLMz: even fine-grained leucomonzogranite.; Ap: apatite, Zrn: zircon, Fe-Mg: ferromagnesian; *Almeida et al. (2006); ${ }^{* *}$ Average 2000 points per thin section. $(\mathrm{x})=$ Minerals present in the thin section, but not detected in the count; $(-)=$ minerals absent on the thin section. 
EmLMz has some amphibole (Tab. 1) and contains evidence of pseudomorphosed pyroxene, which suggest that this facies derived from less evolved melts than the LmLMz. In the latter, chloritized biotite is the main mafic mineral (Fig. 3F), aggregates of calcic plagioclase with mafic minerals are not found and the plagioclase is more sodic and less altered than in previous facies. Muscovite and fluorite, as well as granophyric textures are relatively common in this facies. The fLMz facies presents fine-grained equigranular hypidiomorphic to xenomorphic texture. Biotite crystals are rare and often altered to chlorite.

\section{MINERAL CHEMISTRY}

\section{Analytical Methods}

Seven representative samples of the main facies of the Bannach pluton were selected for mineral chemistry analysis based on previous studies (Almeida et al. 2006): GC (ADR136i), cBAMz (ADR-136C), cABMz (ADR-55A), cLMz (ADR-32B and ADR-32B-1) and LmLMz (ADR-140 and ADR-140-1). Only samples with available whole-rock chemical compositions were selected for mineral chemistry.

The analyzed minerals were amphibole, biotite, plagioclase, titanite, ilmenite, and magnetite. Initially, polished thin sections of the selected samples were carbon coated and then submitted to textural studies and semiquantitative chemical analysis by energy dispersive spectroscopy (EDS) in the Zeiss SIGMA-VP of the Microanalyses Laboratory of the Geosciences Institute of the Federal University of Pará. These data were used to select crystals for wavelength dispersive spectroscopy (WDS) quantitative analysis using a JEOL JXA-8230 microprobe at the same laboratory. WDS analysis were performed under the following operating conditions: a column accelerating voltage of $15 \mathrm{kV}$; a current of $20 \mathrm{nA}$; analysis time of $20 \mathrm{~s}$ to $40 \mathrm{~s}$; and a beam diameter of $10 \mu \mathrm{m}$ for silicates and $5 \mu \mathrm{m}$ for oxides. Matrix effects were corrected by the ZAF method. The crystals used for the analysis were TAP for $\mathrm{Na}, \mathrm{Si}, \mathrm{Al}$, and $\mathrm{Mg}$; PETJ for $\mathrm{Cr}, \mathrm{Ca}, \mathrm{K}$ and $\mathrm{Sr}$; LIFH for $\mathrm{Ni}, \mathrm{Fe}, \mathrm{Mn}, \mathrm{Ba}$, and Ti; PETH for $\mathrm{Cl}$ and V; and LDE1 for F. The standards used for instrument calibration were orthoclase (Si and $\mathrm{K})$, anorthite $(\mathrm{Al})$, wollastonite $(\mathrm{Ca})$, $\mathrm{Fe}_{3} \mathrm{O}_{4}(\mathrm{Fe})$, diopside $(\mathrm{Mg})$, sodalite $(\mathrm{Na}$ and $\mathrm{Cl})$, rutile (Ti), rhodonite $(\mathrm{Mn})$, vanadium $(\mathrm{V}), \mathrm{Cr}_{2} \mathrm{O}_{3}(\mathrm{Cr})$ and biotite $(\mathrm{F})$.

\section{Amphibole}

Amphibole structural formula was calculated based on 23 oxygen atoms, according to the methods of Leake et al. (1997) and Hawthorne et al. (2012). The cations were collected into a set of 13 cations minus $\mathrm{Ca}, \mathrm{Na}$, and $\mathrm{K}$ (13-CNK) (Tab. 2) and 15 cations minus $\mathrm{Na}$ and $\mathrm{K}(15-\mathrm{NK})$ for amphiboles of the Fe-Mg-Mn-Li group (Suppl. Tab. A1). The $\mathrm{Fe}^{+3} / \mathrm{Fe}^{+2}$ ratios were estimated based on charge balancing (Schumacher 1997).

Calcic amphibole of CG and part of the amphiboles of the cBAMz facies are classified as ferro-hornblende (Leake et al. 1997), with $\mathrm{Mg} /\left(\mathrm{Mg}+\mathrm{Fe}^{2+}\right)$ ratios ranging from 0.34 to 0.46 (Fig. 4A). In the other facies, ferro-edenite compositions are

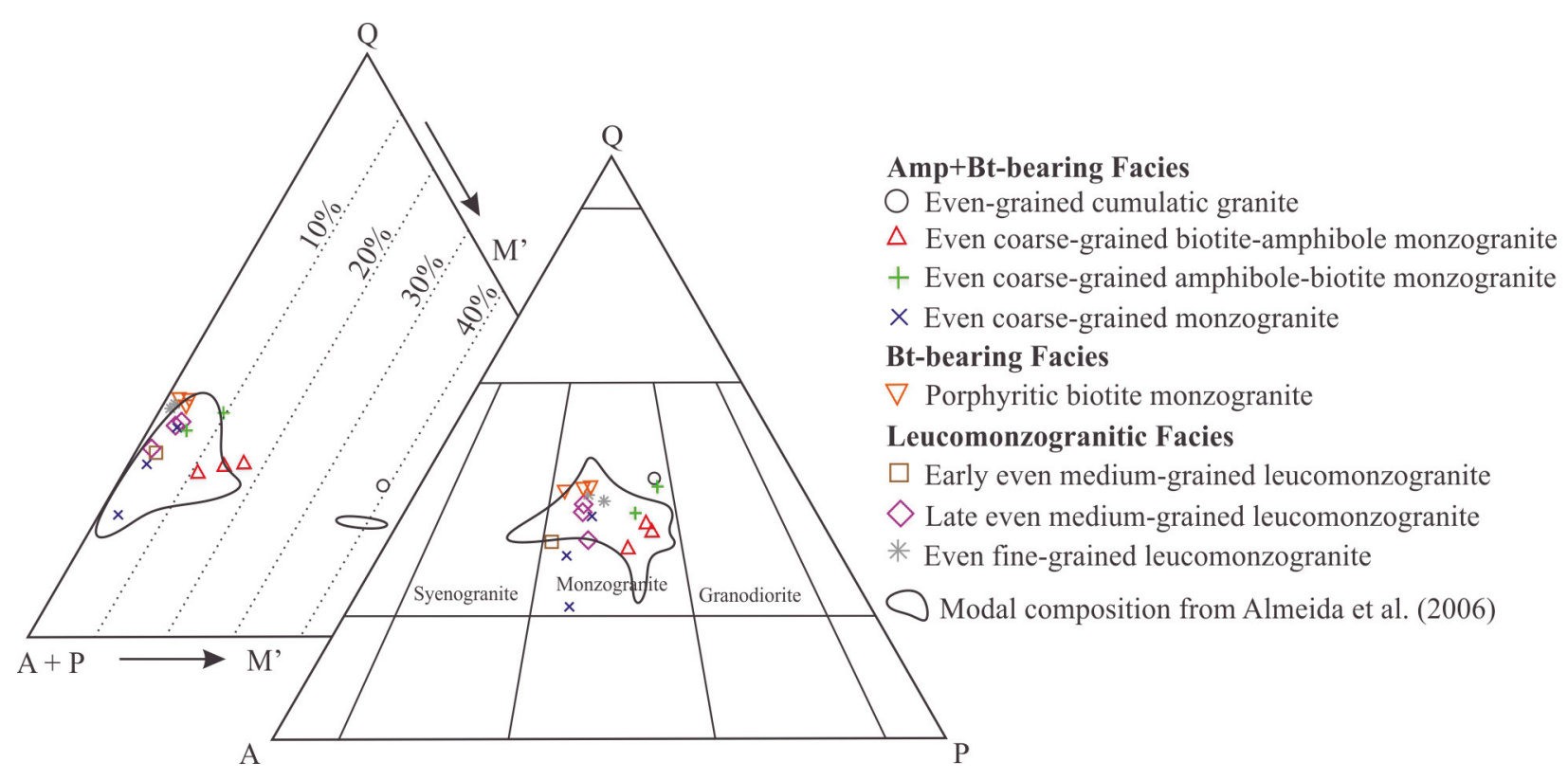

A: Alkali feldspar; P: Plagioclase; Q: Quartz; M’: M - (apatite + muscovite + carbonates). The field of the previously analyzed samples (Almeida et al. 2006) is plotted for comparison.

Figure 2. QAP and Q-A + P-M' (Le Maitre et al. 2002) modal diagrams for the different facies of Bannach Granite. 

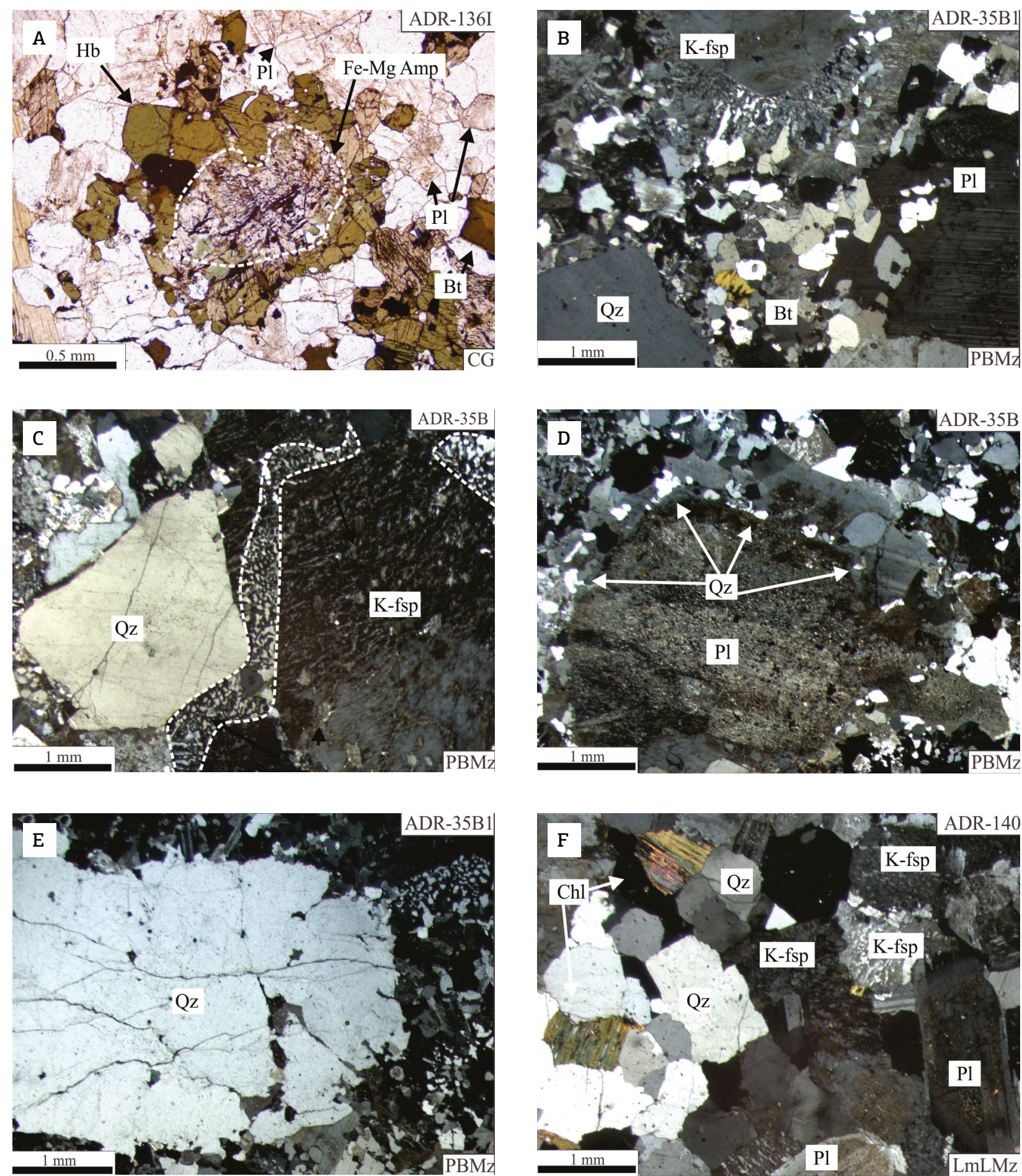

$\mathrm{Qz}$ = quartz; $\mathrm{Pl}$ - plagioclase; $\mathrm{K}$-fsp = potassic feldspar; $\mathrm{Bt}=$ biotite; $\mathrm{Hb}=$ hornblende; $\mathrm{Chl}=$ Chlorite; Fe-Mg Amp = ferromagnesian (clinopyroxene replacemente).

Figure 3. Textural aspects of the Bannach Granite: (A) mafic minerals aggregates associated with partially altered plagioclase. Note ferromagnesian amphibole derived from clinopyroxene (dashed white lines) enveloped by hornblende; (B) quartz, plagioclase and alkali feldspar phenocrysts immersed in a fine-grained quartz-feldspar matrix with dispersed biotite lamellae; (C) quartz - K-feldspar granophyric intergrowths (dashed white lines); (D) plagioclase phenocryst showing an altered calcic core and a preserved sodic rim. Fine-grained quartz included in the border between the two zones; (E) embayed quartz phenocryst immersed in a matrix with granophyric intergrowths; $(F)$ equigranular medium-grained hypidiomorphic texture of LmLMz facies. Note biotite chloritized crystals. Photomicrographs in parallel nicols (A) and crossed nicols (B, C, D, E, F). 
Table 2. Representative electron microprobe analyses of calcic amphiboles of the Bannach Granite.

\begin{tabular}{|c|c|c|c|c|c|c|c|c|c|c|}
\hline \multirow{3}{*}{$\begin{array}{l}\text { Facies } \\
\text { Sample } \\
\text { Analyses }\end{array}$} & \multicolumn{5}{|c|}{ CG } & \multicolumn{5}{|c|}{ cBAMz } \\
\hline & \multicolumn{5}{|c|}{ ADR-136I } & \multicolumn{5}{|c|}{ ADR-136C } \\
\hline & C3_1.10 & C3_1.8 & C3_2.8 & C3_2.10 & C4_1.5 & C3_1.2 & C3_1.5 & C6_1.3 & C6_1.1 & C2_2.5 \\
\hline \multirow{2}{*}{ Type } & Fe-Hb & Fe-Hb & $\mathrm{Fe}-\mathrm{Hb}$ & Fe-Hb & $\mathrm{Fe}-\mathrm{Hb}$ & Fe-Edn & Fe-Edn & $\mathrm{Fe}-\mathrm{Hb}$ & Fe-Edn & Hs \\
\hline & core & rim & core & rim & core & core & rim & core & rim & rim \\
\hline $\mathrm{SiO}_{2}(\mathrm{wt} \%)$ & 43,76 & 43,88 & 43,59 & 43,11 & 43,30 & 42,77 & 42,04 & 42,52 & 41,65 & 40,89 \\
\hline $\mathrm{TiO}_{2}$ & 1,78 & 1,58 & 1,78 & 1,40 & 1,85 & 2,01 & 1,75 & 1,98 & 1,64 & 1,49 \\
\hline $\mathrm{Al}_{2} \mathrm{O}_{3}$ & 7,47 & 7,27 & 7,36 & 6,86 & 7,55 & 7,87 & 7,99 & 7,88 & 8,36 & 8,58 \\
\hline $\mathrm{FeO}$ & 22,95 & 23,49 & 23,70 & 24,46 & 23,27 & 24,46 & 26,78 & 25,66 & 27,05 & 28,42 \\
\hline $\mathrm{MnO}$ & 0,69 & 0,85 & 0,78 & 0,73 & 0,81 & 0,62 & 0,68 & 0,68 & 0,66 & 0,68 \\
\hline $\mathrm{MgO}$ & 7,94 & 7,53 & 7,22 & 6,69 & 7,06 & 6,50 & 4,81 & 5,78 & 4,68 & 3,85 \\
\hline $\mathrm{CaO}$ & 10,20 & 10,01 & 10,00 & 10,26 & 10,15 & 10,31 & 10,38 & 10,04 & 10,27 & 10,32 \\
\hline $\mathrm{Na}_{2} \mathrm{O}$ & 1,87 & 2,01 & 1,99 & 1,75 & 1,92 & 1,88 & 1,86 & 1,96 & 1,89 & 1,82 \\
\hline $\mathrm{K}_{2} \mathrm{O}$ & 1,06 & 0,94 & 1,02 & 0,97 & 1,06 & 1,18 & 1,13 & 1,11 & 1,20 & 1,24 \\
\hline $\mathrm{F}$ & 0,53 & 0,48 & 0,45 & 0,46 & 0,34 & 0,61 & 0,41 & 0,45 & 0,47 & 0,46 \\
\hline $\mathrm{Cl}$ & 0,15 & 0,17 & 0,14 & 0,15 & 0,17 & 0,19 & 0,21 & 0,21 & 0,26 & 0,17 \\
\hline$(\mathrm{OH})$ & 1,87 & 1,96 & 2,20 & 3,33 & 2,67 & 1,78 & 1,88 & 1,80 & 2,08 & 2,18 \\
\hline Subtotal: & 100,3 & 100,2 & 100,2 & 100,2 & 100,15 & 100,2 & 99,9 & 100,1 & 100,2 & 100,1 \\
\hline $\mathrm{O}-\mathrm{F}-\mathrm{Cl}$ & 0,26 & 0,24 & 0,22 & 0,23 & 0,18 & 0,30 & 0,22 & 0,24 & 0,26 & 0,23 \\
\hline Total & 100,00 & 99,93 & 100,00 & 99,95 & 99,96 & 99,88 & 99,69 & 99,83 & 99,96 & 99,87 \\
\hline \multicolumn{11}{|c|}{ Number of cations per formula unit based on twenty three oxygen atoms } \\
\hline $\mathrm{Si}$ & 6,653 & 6,693 & 6,680 & 6,728 & 6,681 & 6,601 & 6,592 & 6,585 & 6,532 & 6,466 \\
\hline $\mathrm{Al}^{\mathrm{IV}}$ & 1,339 & 1,307 & 1,320 & 1,261 & 1,319 & 1,399 & 1,408 & 1,415 & 1,468 & 1,534 \\
\hline $\mathrm{Ti}^{+4}$ & 0,008 & 0,000 & 0,000 & 0,011 & 0,000 & 0,000 & 0,000 & 0,000 & 0,000 & 0,000 \\
\hline Sum T & 8,000 & 8,000 & 8,000 & 8,000 & 8,000 & 8,000 & 8,000 & 8,000 & 8,000 & 8,000 \\
\hline $\mathrm{Al}^{\mathrm{VI}}$ & 0,000 & 0,001 & 0,009 & 0,000 & 0,053 & 0,033 & 0,069 & 0,024 & 0,077 & 0,065 \\
\hline $\mathrm{Ti}^{+3}$ & 0,195 & 0,181 & 0,205 & 0,153 & 0,214 & 0,233 & 0,207 & 0,230 & 0,194 & 0,178 \\
\hline $\mathrm{Fe}^{+3}$ & 0,873 & 0,895 & 0,828 & 0,800 & 0,700 & 0,696 & 0,646 & 0,790 & 0,738 & 0,807 \\
\hline $\mathrm{Fe}^{+2}$ & 2,045 & 2,101 & 2,208 & 2,392 & 2,303 & 2,461 & 2,865 & 2,533 & 2,809 & 2,951 \\
\hline $\mathrm{Mn}$ & 0,088 & 0,110 & 0,101 & 0,097 & 0,106 & 0,081 & 0,090 & 0,089 & 0,087 & 0,091 \\
\hline $\mathrm{Mg}$ & 1,799 & 1,712 & 1,649 & 1,557 & 1,623 & 1,496 & 1,123 & 1,334 & 1,095 & 0,908 \\
\hline Sum C & 5,000 & 5,000 & 5,000 & 5,000 & 5,000 & 5,000 & 5,000 & 5,000 & 5,000 & 5,000 \\
\hline $\mathrm{Ca}$ & 1,661 & 1,637 & 1,642 & 1,715 & 1,678 & 1,704 & 1,744 & 1,666 & 1,725 & 1,749 \\
\hline $\mathrm{Na}$ & 0,339 & 0,363 & 0,358 & 0,285 & 0,322 & 0,296 & 0,256 & 0,334 & 0,275 & 0,251 \\
\hline Sum B & 2,000 & 2,000 & 2,000 & 2,000 & 2,000 & 2,000 & 2,000 & 2,000 & 2,000 & 2,000 \\
\hline $\mathrm{Na}$ & 0,211 & 0,230 & 0,232 & 0,245 & 0,252 & 0,268 & 0,310 & 0,255 & 0,301 & 0,307 \\
\hline $\mathrm{K}$ & 0,205 & 0,182 & 0,199 & 0,194 & 0,208 & 0,231 & 0,226 & 0,220 & 0,240 & 0,251 \\
\hline Sum A & 0,416 & 0,412 & 0,432 & 0,439 & 0,459 & 0,499 & 0,536 & 0,475 & 0,541 & 0,557 \\
\hline $\mathrm{Al}_{\text {total }}$ & 1,339 & 1,308 & 1,329 & 1,261 & 1,372 & 1,431 & 1,477 & 1,439 & 1,545 & 1,598 \\
\hline $\mathrm{Fe} /(\mathrm{Fe}+\mathrm{Mg})$ & 0,532 & 0,551 & 0,573 & 0,606 & 0,606 & 0,622 & 0,718 & 0,655 & 0,719 & 0,719 \\
\hline $\mathrm{Mg} /(\mathrm{Mg}+\mathrm{Fe})$ & 0,468 & 0,449 & 0,427 & 0,394 & 0,394 & 0,378 & 0,282 & 0,345 & 0,281 & 0,281 \\
\hline
\end{tabular}


Table 2. Continuation.

\begin{tabular}{|c|c|c|c|c|c|c|c|c|c|}
\hline \multirow{3}{*}{$\begin{array}{l}\text { Facies } \\
\text { Sample } \\
\text { Analyses }\end{array}$} & \multicolumn{5}{|c|}{ cABMz } & \multicolumn{4}{|c|}{ cLMz } \\
\hline & \multicolumn{5}{|c|}{ ADR-55A } & \multicolumn{4}{|c|}{ ADR-32B1 } \\
\hline & C1_1.3 & C1_1.5 & C2_1.2 & C2_1.5 & C2_1.3 & C2_1.5 & C2_1.7 & C2_1.4 & C2_1.8 \\
\hline \multirow{2}{*}{ Type } & Fe-Edn & Hs & Fe-Edn & Fe-Edn & Fe-Edn & Fe-Edn & Fe-Edn & Fe-Edn & Fe-Edn \\
\hline & core & rim & core & rim & core & core & rim & core & rim \\
\hline $\mathrm{SiO}_{2}(\mathrm{wt} \%)$ & 41,90 & 40,35 & 41,75 & 41,45 & 41,85 & 43,04 & 42,22 & 43,00 & 42,69 \\
\hline $\mathrm{TiO}_{2}$ & 1,46 & 1,35 & 1,04 & 1,79 & 1,89 & 1,97 & 1,81 & 2,03 & 1,87 \\
\hline $\mathrm{Al}_{2} \mathrm{O}_{3}$ & 7,74 & 8,72 & 7,62 & 7,72 & 7,78 & 7,54 & 7,72 & 7,56 & 7,57 \\
\hline $\mathrm{FeO}$ & 28,60 & 29,28 & 27,80 & 28,53 & 26,74 & 24,11 & 26,58 & 25,14 & 26,12 \\
\hline $\mathrm{MnO}$ & 0,83 & 0,84 & 0,72 & 0,91 & 0,80 & 0,72 & 0,96 & 0,69 & 0,87 \\
\hline $\mathrm{MgO}$ & 3,82 & 3,16 & 4,45 & 3,60 & 4,91 & 6,91 & 5,09 & 6,43 & 5,58 \\
\hline $\mathrm{CaO}$ & 10,22 & 10,28 & 10,44 & 10,10 & 9,90 & 10,16 & 10,06 & 10,29 & 10,23 \\
\hline $\mathrm{Na}_{2} \mathrm{O}$ & 1,95 & 1,96 & 1,81 & 1,96 & 2,13 & 2,04 & 2,07 & 2,05 & 2,04 \\
\hline $\mathrm{K}_{2} \mathrm{O}$ & 1,19 & 1,38 & 1,30 & 1,19 & 1,16 & 1,15 & 1,15 & 1,20 & 1,16 \\
\hline $\mathrm{F}$ & 0,60 & 0,65 & 0,66 & 0,60 & 0,76 & 0,98 & 0,78 & 0,83 & 0,97 \\
\hline $\mathrm{Cl}$ & 0,24 & 0,35 & 0,23 & 0,22 & 0,24 & 0,13 & 0,19 & 0,14 & 0,16 \\
\hline (OH) & 1,72 & 1,96 & 2,25 & 2,11 & 1,81 & 1,36 & 1,54 & 0,89 & 1,15 \\
\hline Subtotal: & 100,3 & 100,28 & 100,077 & 100,2 & 100,0 & 100,1 & 100,2 & 100,3 & 100,4 \\
\hline $\mathrm{O}-\mathrm{F}-\mathrm{Cl}$ & 0,31 & 0,35 & 0,3305176 & 0,30 & 0,37 & 0,44 & 0,37 & 0,38 & 0,44 \\
\hline Total & 99,96 & 99,93 & 99,746482 & 99,89 & 99,59 & 99,67 & 99,81 & 99,88 & 99,95 \\
\hline \multicolumn{10}{|c|}{ Number of cations per formula unit based on twenty three oxygen atoms } \\
\hline $\mathrm{Si}$ & 6,607 & 6,434 & 6,619 & 6,580 & 6,571 & 6,623 & 6,588 & 6,606 & 6,615 \\
\hline $\mathrm{Al}^{\mathrm{IV}}$ & 1,393 & 1,566 & 1,381 & 1,420 & 1,429 & 1,366 & 1,412 & 1,369 & 1,382 \\
\hline $\mathrm{Ti}^{+4}$ & 0,000 & 0,000 & 0,000 & 0,000 & 0,000 & 0,010 & 0,000 & 0,025 & 0,003 \\
\hline Sum T & 8,000 & 8,000 & 8,000 & 8,000 & 8,000 & 8,000 & 8,000 & 8,000 & 8,000 \\
\hline $\mathrm{Al}^{\mathrm{VI}}$ & 0,046 & 0,072 & 0,043 & 0,024 & 0,010 & 0,000 & 0,008 & 0,000 & 0,000 \\
\hline $\mathrm{Ti}^{+3}$ & 0,174 & 0,162 & 0,124 & 0,214 & 0,223 & 0,218 & 0,213 & 0,209 & 0,215 \\
\hline $\mathrm{Fe}^{+3}$ & 0,713 & 0,770 & 0,725 & 0,689 & 0,765 & 0,749 & 0,761 & 0,718 & 0,713 \\
\hline $\mathrm{Fe}^{+2}$ & 3,059 & 3,133 & 2,960 & 3,098 & 2,746 & 2,353 & 2,707 & 2,512 & 2,670 \\
\hline $\mathrm{Mn}$ & 0,111 & 0,113 & 0,097 & 0,122 & 0,106 & 0,093 & 0,127 & 0,089 & 0,114 \\
\hline $\mathrm{Mg}$ & 0,898 & 0,750 & 1,051 & 0,853 & 1,150 & 1,586 & 1,184 & 1,473 & 1,288 \\
\hline Sum C & 5,000 & 5,000 & 5,000 & 5,000 & 5,000 & 5,000 & 5,000 & 5,000 & 5,000 \\
\hline $\mathrm{Ca}$ & 1,726 & 1,756 & 1,773 & 1,717 & 1,665 & 1,674 & 1,681 & 1,693 & 1,698 \\
\hline $\mathrm{Na}$ & 0,274 & 0,244 & 0,227 & 0,283 & 0,335 & 0,326 & 0,319 & 0,307 & 0,302 \\
\hline Sum B & 2,000 & 2,000 & 2,000 & 2,000 & 2,000 & 2,000 & 2,000 & 2,000 & 2,000 \\
\hline $\mathrm{Na}$ & 0,323 & 0,362 & 0,329 & 0,321 & 0,312 & 0,282 & 0,308 & 0,304 & 0,312 \\
\hline K & 0,238 & 0,281 & 0,263 & 0,240 & 0,232 & 0,225 & 0,228 & 0,236 & 0,229 \\
\hline Sum A & 0,561 & 0,643 & 0,591 & 0,562 & 0,544 & 0,507 & 0,536 & 0,540 & 0,541 \\
\hline $\mathrm{Al}_{\text {total }}$ & 1,439 & 1,638 & 1,424 & 1,444 & 1,439 & 1,366 & 1,420 & 1,369 & 1,382 \\
\hline $\mathrm{Fe} /(\mathrm{Fe}+\mathrm{Mg})$ & 0,773 & 0,807 & 0,784 & 0,784 & 0,705 & 0,597 & 0,696 & 0,630 & 0,675 \\
\hline $\mathrm{Mg} /(\mathrm{Mg}+\mathrm{Fe})$ & 0,227 & 0,193 & 0,216 & 0,216 & 0,295 & 0,403 & 0,304 & 0,370 & 0,325 \\
\hline
\end{tabular}

CG: even-grained cumulatic granite; cBAMz: even coarse-grained biotite-amphibole monzogranite; cABMz: even coarse-grained amphibole-biotite monzogranite; cLMz: even coarse-grained leucomonzogranite; Fe-Edn: ferro-edenite; Hs: hastingsite. 


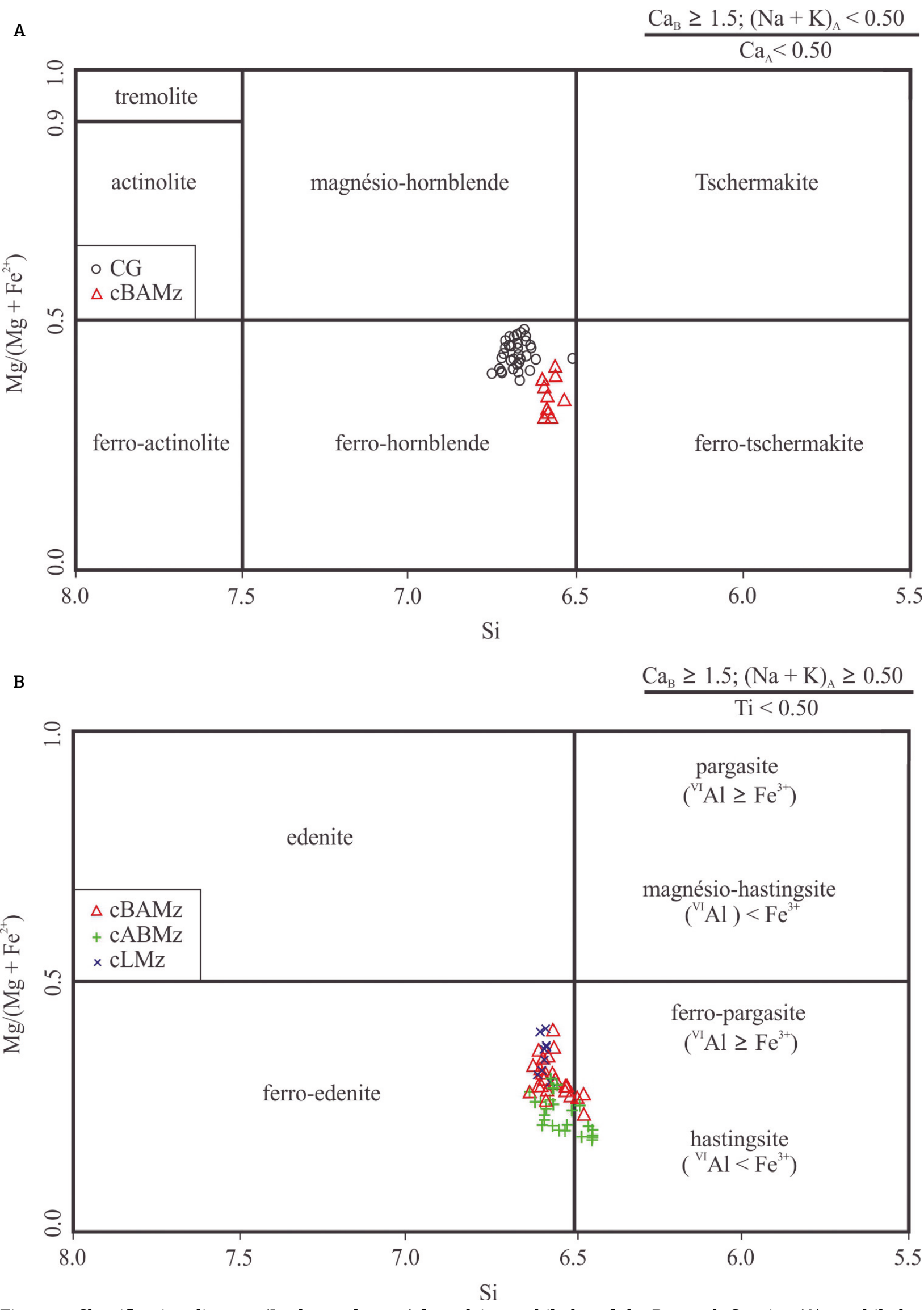

Figure 4. Classification diagram (Leake et al. 1997) for calcic amphiboles of the Bannach Granite: (A) amphiboles of the $\mathrm{Ca}_{\mathrm{B}} \geq 1.50 ;(\mathrm{Na}+\mathrm{K})_{\mathrm{A}}<0.50 ; \mathrm{Ca}_{\mathrm{A}}<0.50$ group; $(\mathrm{B})$ amphiboles of the $\mathrm{Ca}_{\mathrm{B}} \geq 1.50 ;(\mathrm{Na}+\mathrm{K})_{A} \geq 0.50 ; \mathrm{Ti}<0.50$ group. 
dominant, whereas hastingsite (Leake et al. 1997) with $\mathrm{Mg} /$ $(\mathrm{Mg}+\mathrm{Fe})$ ratios varying from 0.19 to 0.37 (Fig. 4B) is subordinate. In the Bannach Granite, amphibole evolution from GC to $\mathrm{cBAMz}$ and $\mathrm{cABMz}$ is marked by a decrease of $\mathrm{Si}$ and an increase of alkalis $(\mathrm{Na}+\mathrm{K})$ and $\mathrm{Fe} /(\mathrm{Fe}+\mathrm{Mg})$ ratios (Tab. 2). On the other hand, the composition of the calcic amphibole from cLMz facies overlaps with that of cBAMz facies (Fig. $4 \mathrm{~B})$. Usually, the core and rim compositions of individual crystals also show significant variations. The core is slightly enriched in $\mathrm{Si}$ and $\mathrm{Mg}$, and the rim is more alkaline and ferrous, sometimes with a transition from ferro-edenite to hastingsite (Tab. 2). In the analyzed amphiboles, $\mathrm{OH}$ and $\mathrm{F}$ contents are higher than $\mathrm{Cl}$ contents. Moreover, in the cLMz facies, $\mathrm{F}$ contents are higher when compared to other facies (Tab. 2).

Amphibole of Fe-Mg-Mn-Li group is common in CG, cBAMz, and cABMz facies and rare or absent in the other facies. It is classified as cummingtonite-grunerite (Leake et al. 1997), based on composition and oblique extinction in thin section, and has $\mathrm{Mg} /\left(\mathrm{Mg}+\mathrm{Fe}^{2+}\right)$ ratios ranging from 0.43 to $\sim 0.55$ (Fig. 5).

\section{Biotite}

The structural formula was calculated on the basis of 22 atoms of oxygens and it was assumed that all iron is in the $\mathrm{Fe}^{2+}$ state (Tab. 3). The biotite crystals of the Bannach Granite are ferroan and, in the LmLMz facies, they approach annite in composition (Fig. 6A). They are divided into three groups: the first group is represented by the biotite of CG facies that has $\mathrm{Fe} /(\mathrm{Fe}+\mathrm{Mg})$ ratio varying from 0.62 to 0.67 . In the second, this ratio ranges from 0.72 to 0.80 and it comprises biotites of cBAMz, cABMz and cLMz facies. Lastly, the biotite of the third group has near annite composition $[\mathrm{Fe} /(\mathrm{Fe}+\mathrm{Mg})$ ratios of 0.89 to $0.91 ; \mathrm{LmLMz}$ facies]. The $\mathrm{Al}^{\mathrm{IV}}$ in biotite is almost constant in all facies (2.22 to 2.35) except for the biotite of $\mathrm{LmLMz}$ facies that has relatively higher $\mathrm{Al}^{\mathrm{VI}}$ and total $\mathrm{Al}$ values (Tab. 3). As a result, in the $\mathrm{Mg} \times$ Alt diagram (Fig. 6B; fields of Nachit et al. 1985) biotite of LmLMz facies plot in the transition between alkaline and sub-alkaline fields whereas those of other facies are limited to the alkaline field. In the $\left(\mathrm{FeO}_{\mathrm{t}}+\mathrm{MnO}\right)-\left(10 * \mathrm{TiO}_{2}\right)-\mathrm{MgO}$ ternary diagram (Fig. 6C; fields of Nachit 1994), they plot in the magmatic primary biotite field. As noted for amphibole crystals, biotite grains of all facies have fluorine contents dominant over chlorine. However, in the cLMz, this contrast is less marked. The highest F contents is recorded in cABMz and, locally, in the $\mathrm{LmLMz}$ facies (Tab. 3).

$\left(\mathrm{Ca}+\mathrm{Na}_{\mathrm{B}}\right)<1.00 ;\left(\mathrm{Mg}, \mathrm{Fe}^{2+}, \mathrm{Mn}, \mathrm{Li}\right)_{\mathrm{B}} \geq 1.00$

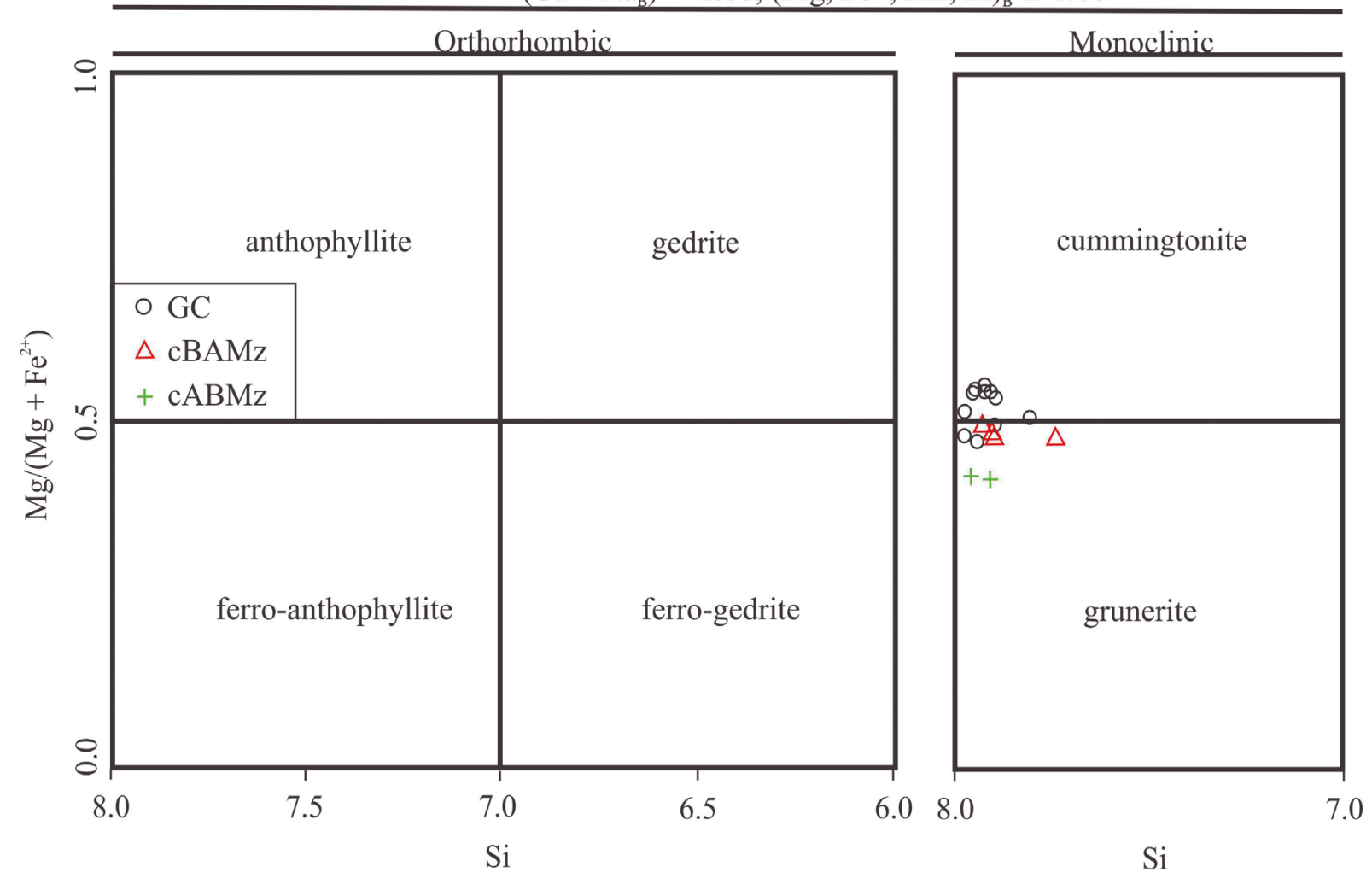

Figure 5. Classification diagram for the Mg-Fe-Mn-Li amphiboles (Leake et al. 1997) of the Bannach Granite. 
Table 3. Representative electron microprobe analyses of biotite of the Bannach Granite.

\begin{tabular}{|c|c|c|c|c|c|c|c|c|c|c|}
\hline \multirow{3}{*}{$\begin{array}{l}\text { Facies } \\
\text { Sample } \\
\text { Analyses }\end{array}$} & \multicolumn{4}{|c|}{ CG } & \multicolumn{4}{|c|}{ cBAMz } & \multicolumn{2}{|c|}{ cABMz } \\
\hline & \multicolumn{4}{|c|}{ ADR-136I } & \multicolumn{4}{|c|}{ ADR-136C } & \multicolumn{2}{|c|}{ ADR-55A } \\
\hline & C3_1.1 & C4_1.2 & C4_2.1 & C5_1.2 & C1_1.2 & C1_1.5 & C5_1.1 & C5_1.4 & C1_1.1 & C1_2.1 \\
\hline $\mathrm{SiO}_{2}(\mathrm{wt} \%)$ & 36,36 & 36,09 & 36,30 & 36,91 & 35,39 & 35,37 & 35,48 & 35,83 & 35,32 & 35,24 \\
\hline $\mathrm{TiO}_{2}$ & 3,47 & 4,00 & 3,67 & 3,75 & 4,20 & 4,11 & 4,52 & 4,06 & 3,88 & 4,15 \\
\hline $\mathrm{Al}_{2} \mathrm{O}_{3}$ & 12,45 & 12,00 & 12,21 & 12,08 & 12,47 & 12,39 & 12,55 & 12,31 & 12,32 & 12,23 \\
\hline $\mathrm{FeO}$ & 26,13 & 26,16 & 26,44 & 25,45 & 29,88 & 29,59 & 29,04 & 28,34 & 30,85 & 30,41 \\
\hline $\mathrm{MnO}$ & 0,35 & 0,32 & 0,28 & 0,34 & 0,35 & 0,33 & 0,36 & 0,31 & 0,30 & 0,47 \\
\hline $\mathrm{MgO}$ & 8,05 & 8,07 & 7,19 & 8,61 & 4,70 & 4,99 & 5,17 & 6,07 & 4,10 & 4,36 \\
\hline $\mathrm{CaO}$ & 0,00 & 0,03 & 0,03 & 0,00 & 0,04 & 0,04 & 0,02 & 0,00 & 0,02 & 0,02 \\
\hline $\mathrm{Na} 2 \mathrm{O}$ & 0,03 & 0,08 & 0,03 & 0,09 & 0,05 & 0,08 & 0,05 & 0,08 & 0,08 & 0,08 \\
\hline K2O & 9,26 & 9,24 & 9,17 & 9,28 & 9,01 & 8,92 & 9,23 & 9,25 & 9,10 & 9,16 \\
\hline $\mathrm{F}$ & 0,64 & 0,53 & 0,57 & 0,63 & 0,29 & 0,26 & 0,45 & 0,69 & 0,95 & 0,79 \\
\hline $\mathrm{Cl}$ & 0,22 & 0,20 & 0,23 & 0,21 & 0,19 & 0,26 & 0,19 & 0,21 & 0,30 & 0,28 \\
\hline$(\mathrm{OH})$ & 3,20 & 3,43 & 3,96 & 2,69 & 3,48 & 3,53 & 2,98 & 2,75 & 3,02 & 3,04 \\
\hline Subtotal: & 100,17 & 100,14 & 100,07 & 100,04 & 100,06 & 99,87 & 100,02 & 99,89 & 100,24 & 100,23 \\
\hline $\mathrm{O}-\mathrm{F}-\mathrm{Cl}$ & 0,32 & 0,27 & 0,29 & 0,31 & 0,16 & 0,17 & 0,23 & 0,34 & 0,47 & 0,40 \\
\hline Total & 99,85 & 99,88 & 99,78 & 99,72 & 99,89 & 99,70 & 99,78 & 99,55 & 99,77 & 99,83 \\
\hline
\end{tabular}

Number of cations per formula unit based on twenty two oxygen atoms

\begin{tabular}{l|l|l|l|l|l|l|l|l|l|l}
\hline $\mathrm{Si}$ & 5,709 & 5,685 & 5,753 & 5,744 & 5,620 & 5,674 & 5,643 & 5,684 & 5,695 & 5,671 \\
\hline $\mathrm{Al}^{\mathrm{IV}}$ & 2,291 & 2,228 & 2,247 & 2,216 & 2,335 & 2,326 & 2,352 & 2,302 & 2,305 & 2,320 \\
\hline $\mathrm{Al}^{\mathrm{VI}}$ & 0,013 & 0,000 & 0,034 & 0,000 & 0,000 & 0,017 & 0,000 & 0,000 & 0,037 & 0,000 \\
\hline $\mathrm{Ti}$ & 0,410 & 0,473 & 0,438 & 0,439 & 0,502 & 0,496 & 0,541 & 0,485 & 0,470 & 0,503 \\
\hline $\mathrm{Fe}$ & 3,431 & 3,447 & 3,504 & 3,313 & 3,968 & 3,970 & 3,863 & 3,760 & 4,160 & 4,093 \\
\hline $\mathrm{Mn}$ & 0,047 & 0,043 & 0,038 & 0,044 & 0,047 & 0,045 & 0,048 & 0,042 & 0,042 & 0,063 \\
\hline $\mathrm{Mg}$ & 1,884 & 1,895 & 1,698 & 1,997 & 1,113 & 1,193 & 1,227 & 1,436 & 0,985 & 1,045 \\
\hline $\mathrm{Ca}$ & 0,000 & 0,005 & 0,005 & 0,001 & 0,006 & 0,007 & 0,003 & 0,000 & 0,004 & 0,003 \\
\hline $\mathrm{Na}$ & 0,010 & 0,023 & 0,010 & 0,026 & 0,017 & 0,025 & 0,015 & 0,024 & 0,024 & 0,025 \\
\hline $\mathrm{K}$ & 1,854 & 1,857 & 1,854 & 1,842 & 1,824 & 1,825 & 1,872 & 1,871 & 1,871 & 1,880 \\
\hline $\mathrm{OH}$ & 3,621 & 3,684 & 3,656 & 3,635 & 3,805 & 3,798 & 3,723 & 3,600 & 3,435 & 3,520 \\
\hline $\mathrm{F}$ & 0,320 & 0,263 & 0,283 & 0,309 & 0,143 & 0,130 & 0,227 & 0,345 & 0,483 & 0,404 \\
\hline $\mathrm{Cl}$ & 0,059 & 0,053 & 0,060 & 0,056 & 0,052 & 0,071 & 0,050 & 0,055 & 0,082 & 0,076 \\
\hline $\mathrm{Al}$ Total & 2,304 & 2,228 & 2,281 & 2,216 & 2,335 & 2,344 & 2,352 & 2,302 & 2,342 & 2,320 \\
\hline $\mathrm{Fe} / \mathrm{Fe}+\mathrm{Mg}$ & 0,646 & 0,645 & 0,674 & 0,624 & 0,781 & 0,769 & 0,759 & 0,724 & 0,808 & 0,797 \\
\hline & & & & & & & & $\mathrm{Continue} .$. \\
& & & & & & & \\
\hline
\end{tabular}


Table 3. Continuation.

\begin{tabular}{|c|c|c|c|c|c|c|c|c|c|c|}
\hline \multirow{3}{*}{$\begin{array}{l}\text { Facies } \\
\text { Sample } \\
\text { Analyses }\end{array}$} & \multirow{2}{*}{\multicolumn{2}{|c|}{$\begin{array}{c}\text { cABMz } \\
\text { ADR-55A }\end{array}$}} & \multicolumn{4}{|c|}{ cLMz } & \multicolumn{4}{|c|}{ LmLMz } \\
\hline & & & \multicolumn{2}{|c|}{ ADR-32B } & \multicolumn{2}{|c|}{ ADR-32B1 } & \multicolumn{2}{|c|}{ ADR-140 } & \multicolumn{2}{|c|}{ ADR-140-1 } \\
\hline & C2_1.2 & C2_1.3 & C2_1.1 & C2_1.4 & C2_2.2 & C2_4.3 & C1_1.1 & C3_1.3 & C1_1.1 & C3_1.1 \\
\hline $\mathrm{SiO}_{2}(\mathrm{wt} \%)$ & 35,17 & 35,28 & 35,48 & 36,07 & 36,03 & 35,44 & 35,66 & 35,97 & 35,30 & 35,35 \\
\hline $\mathrm{TiO}_{2}$ & 3,77 & 4,09 & 3,56 & 3,93 & 3,47 & 3,64 & 3,78 & 2,77 & 3,71 & 3,21 \\
\hline $\mathrm{Al}_{2} \mathrm{O}_{3}$ & 12,23 & 12,16 & 12,66 & 12,24 & 12,29 & 12,66 & 15,48 & 16,21 & 14,40 & 15,56 \\
\hline $\mathrm{FeO}$ & 31,01 & 30,65 & 29,60 & 29,11 & 29,16 & 29,91 & 28,72 & 29,13 & 30,70 & 29,72 \\
\hline $\mathrm{MnO}$ & 0,41 & 0,39 & 0,52 & 0,50 & 0,56 & 0,30 & 0,40 & 0,62 & 0,43 & 0,47 \\
\hline $\mathrm{MgO}$ & 4,30 & 4,28 & 5,71 & 5,16 & 5,48 & 5,17 & 1,90 & 1,80 & 1,72 & 1,92 \\
\hline $\mathrm{CaO}$ & 0,00 & 0,00 & 0,01 & 0,00 & 0,01 & 0,02 & 0,01 & 0,01 & 0,04 & 0,00 \\
\hline $\mathrm{Na} 2 \mathrm{O}$ & 0,08 & 0,09 & 0,05 & 0,06 & 0,05 & 0,06 & 0,14 & 0,08 & 0,14 & 0,06 \\
\hline $\mathrm{K} 2 \mathrm{O}$ & 8,97 & 9,02 & 8,43 & 9,03 & 9,27 & 8,90 & 9,11 & 9,28 & 9,05 & 9,27 \\
\hline $\mathrm{F}$ & 0,75 & 0,80 & 0,28 & 0,27 & 0,52 & 0,29 & 0,65 & 0,81 & 0,39 & 0,56 \\
\hline $\mathrm{Cl}$ & 0,29 & 0,31 & 0,31 & 0,30 & 0,26 & 0,29 & 0,22 & 0,17 & 0,46 & 0,26 \\
\hline$(\mathrm{OH})$ & 3,12 & 3,08 & 3,56 & 3,29 & 3,10 & 3,32 & 3,60 & 3,33 & 3,73 & 3,77 \\
\hline Subtotal: & 100,09 & 100,14 & 100,16 & 99,97 & 100,20 & 100,00 & 99,65 & 100,18 & 100,05 & 100,14 \\
\hline $\mathrm{O}-\mathrm{F}-\mathrm{Cl}$ & 0,38 & 0,40 & 0,18 & 0,18 & 0,28 & 0,19 & 0,32 & 0,38 & 0,27 & 0,29 \\
\hline Total & 99,71 & 99,73 & 99,97 & 99,78 & 99,93 & 99,81 & 99,33 & 99,80 & 99,78 & 99,85 \\
\hline \multicolumn{11}{|c|}{ Number of cations per formula unit based on twenty two oxygen atoms } \\
\hline $\mathrm{Si}$ & 5,681 & 5,685 & 5,663 & 5,747 & 5,740 & 5,670 & 5,688 & 5,714 & 5,699 & 5,667 \\
\hline $\mathrm{Al}^{\mathrm{IV}}$ & 2,319 & 2,310 & 2,337 & 2,253 & 2,260 & 2,330 & 2,312 & 2,286 & 2,301 & 2,333 \\
\hline $\mathrm{Al}^{\mathrm{VI}}$ & 0,010 & 0,000 & 0,044 & 0,045 & 0,048 & 0,057 & 0,599 & 0,749 & 0,439 & 0,607 \\
\hline $\mathrm{Ti}$ & 0,457 & 0,495 & 0,428 & 0,471 & 0,415 & 0,438 & 0,453 & 0,331 & 0,451 & 0,387 \\
\hline $\mathrm{Fe}$ & 4,188 & 4,130 & 3,950 & 3,879 & 3,886 & 4,002 & 3,832 & 3,870 & 4,145 & 3,984 \\
\hline $\mathrm{Mn}$ & 0,056 & 0,054 & 0,070 & 0,068 & 0,076 & 0,041 & 0,054 & 0,083 & 0,058 & 0,063 \\
\hline $\mathrm{Mg}$ & 1,035 & 1,028 & 1,358 & 1,225 & 1,302 & 1,233 & 0,451 & 0,426 & 0,413 & 0,458 \\
\hline $\mathrm{Ca}$ & 0,000 & 0,000 & 0,002 & 0,000 & 0,002 & 0,003 & 0,001 & 0,001 & 0,006 & 0,000 \\
\hline $\mathrm{Na}$ & 0,024 & 0,027 & 0,014 & 0,018 & 0,015 & 0,017 & 0,042 & 0,024 & 0,044 & 0,019 \\
\hline K & 1,849 & 1,854 & 1,716 & 1,836 & 1,884 & 1,816 & 1,853 & 1,881 & 1,864 & 1,897 \\
\hline $\mathrm{OH}^{*}$ & 3,539 & 3,511 & 3,778 & 3,781 & 3,668 & 3,772 & 3,613 & 3,547 & 3,677 & 3,645 \\
\hline $\mathrm{F}$ & 0,381 & 0,406 & 0,139 & 0,137 & 0,262 & 0,149 & 0,329 & 0,407 & 0,198 & 0,283 \\
\hline $\mathrm{Cl}$ & 0,080 & 0,084 & 0,083 & 0,082 & 0,070 & 0,079 & 0,058 & 0,046 & 0,125 & 0,071 \\
\hline $\mathrm{Al}_{\text {Total }}$ & 2,329 & 2,310 & 2,382 & 2,298 & 2,308 & 2,387 & 2,910 & 3,035 & 2,740 & 2,940 \\
\hline $\mathrm{Fe} / \mathrm{Fe}+\mathrm{Mg}$ & 0,802 & 0,801 & 0,744 & 0,760 & 0,749 & 0,764 & 0,895 & 0,901 & 0,909 & 0,897 \\
\hline
\end{tabular}

CG: even-grained cumulatic granite; cBAMz: even coarse-grained biotite-amphibole monzogranite; cABMz: even coarse-grained amphibole-biotite monzogranite; cLMz: even coarse-grained leucomonzogranite; LmLMz: late even medium-grained leucomonzogranite. 

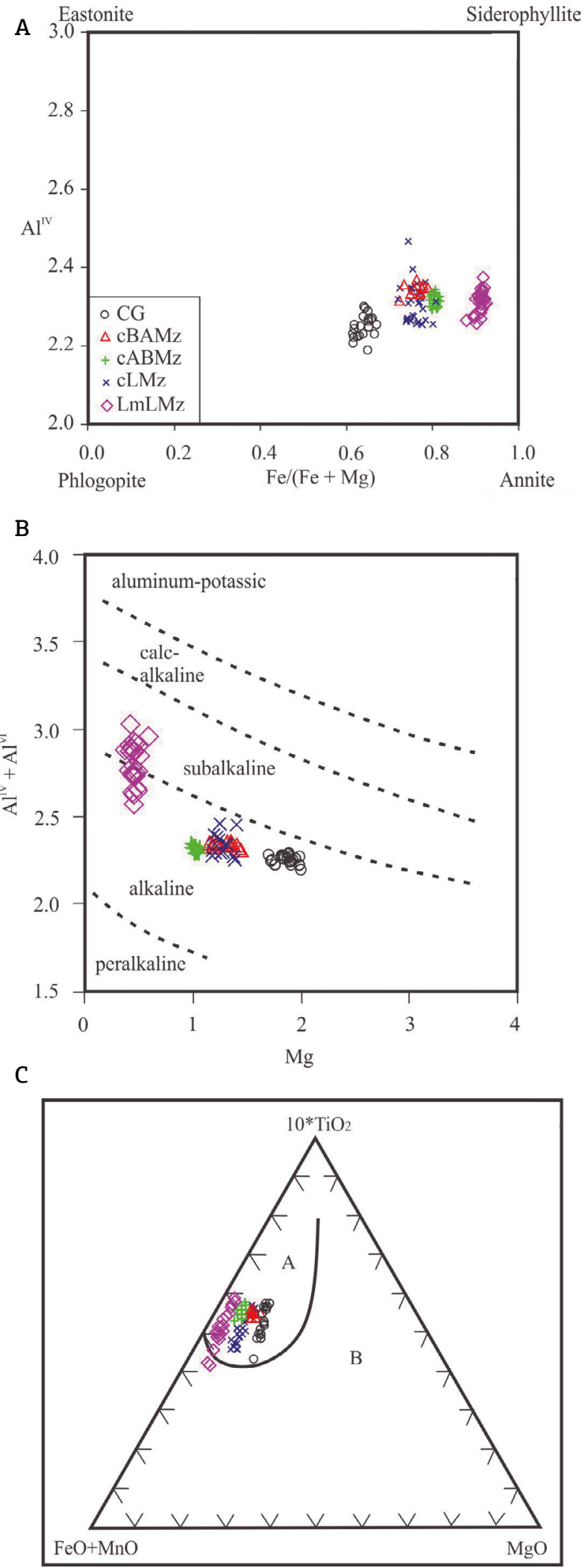

A: primary magmatic biotite; B: reequilibrated magmatic biotite.

Figure 6. Composition of the biotite of the Bannach granite: (A) $\mathrm{Fe} /(\mathrm{Fe}+\mathrm{Mg}) \times \mathrm{Al}^{\mathrm{IV}}$ (Deer et al. 1992) diagram; (B) $\mathrm{Mg} \times$ Alt (Nachit et al. 1985) diagram; (C) $(\mathrm{FeO}+$ $\mathrm{MnO})-\left(10^{*} \mathrm{TiO}_{2}\right)$ - MgO diagram (fields of Nachit 1994).

\section{Plagioclase}

Plagioclase crystals of all varieties of the Bannach Granite show normal zoning and general composition varying from oligoclase to albite (Figs. 7A, 7B, 7C, 7D), sometimes with sodic andesine cores (Tab. 4). The results obtained show that plagioclase composition overlaps with those estimated by petrographic methods for the Bannach Granite (Almeida et al. 2006) and determined in the case of the Jamon Granite (Dall'Agnol 1982, Dall'Agnol et al. 1999b, 1999c). In this study, in the core of early plagioclase aggregates observed in the CG, cBAMz, and cABMz facies, anorthite contents range between $\mathrm{An}_{38}$ and $\mathrm{An}_{23}$ and more sodic oligoclase compositions are observed in plagioclase rims. Similar sodic oligoclase compositions are found in the core of cLMz and LmLMz. In the cLMz and LmLMz facies plagioclase rims have albite composition $\left(\mathrm{An}_{6.1}\right.$ to $\left.\mathrm{An}_{2.8}\right)$.

Assuming a magmatic model similar to that proposed for the Jamon Granite (Dall'Agnol 1982), it can be admitted that the more calcic plagioclase cores of less evolved facies were generated in an initial magmatic stage at high-pressure conditions. In a next step, after rapid magma ascent and fall of pressure, early plagioclase was destabilized and sodic rims started to crystallize. This stage took place simultaneously with the formation of the plagioclase cores of the more evolved facies (pBMz, cLMz, EmLMz, LmLMz, and $\mathrm{fLMz}$ ). The more sodic plagioclase of $\mathrm{LmLMz}$ facies could be derived from a highly differentiated or independent melt, distinct from those which generated the other facies of Bannach Granite (Almeida et al. 2006).

\section{Titanite}

In the Bannach Granite, titanite occurs as subhedral crystals often associated with biotite and opaque minerals. It shows Fe/ $\mathrm{Al}$ ratios between 0.48 and 0.57 which, according to Kowallis et al. (1997), are correlated with titanite from plutonic rocks with quartz (Suppl. Tab. A2). According to Dall'Agnol et al. (1999c), in the Jamon Granite, titanite is stable under relatively oxidizing conditions and its formation is related to a peritetic reaction involving the destabilization of the amphibole in a late magmatic stage. This can also be applied to the crystallization of titanite in the Bannach Granite.

\section{Ilmenite}

Four textural types of ilmenite were reported in the Bannach Granite, which are analogous to those described for the Jamon Granite (Dall'Agnol et al. 1997). The most common is trellis ilmenite ( $\mathrm{T} \mathrm{Ilm}$ ) present in all analyzed facies of the Bannach Granite. It occurs as intergrowths with magnetite (Figs. 8A, 8B) and is resulted from oxidation-exsolution of primary titanomagnetites during or after magmatic crystallization (Buddington \& Lindsley 1964, Haggerty 
1991). In the beginning of the oxi-exsolution process, the T Ilm lamellae are fine (Fig. 8A) and the magnetite forming the intergrowths is relatively enriched in Ti (Suppl. Tab. A4, see C3_2-2 e C1_1-3 analysis). In more advanced stages, the size of ilmenite lamellae increases and Ti contents in the magnetite decrease (Fig. 8B) (Haggerty 1991). Another kind of textural variety is patch ilmenite (P Ilm) corresponding to the irregularly shaped ilmenite that occurs at the rim of crystals with magnetite-T Ilm intergrowths or along the contacts of two distinct oxide crystals (Fig. 8C). The third variety is composite ilmenite (C Ilm), which is associated with magnetite crystals and generally subhedral (Fig. 8D). Finally, individual ilmenite (I Ilm) is subhedral to euhedral (Fig 8E) and occurs dissociated from magnetite. In the Jamon Granite, I Ilm and C Ilm are early crystallized (Dall'Agnol et al. 1997). This is also probably true in the case of Bannach Granite, even if there is no accentuate compositional contrast between the different textural varieties of ilmenite (Suppl. Tab. A3).

\section{Magnetite}

During the slow cooling of a granitic magma, the original crystals of titanomagnetite are affected by oxy-exsolution process and hardly preserve their primary composition (Buddington \& Lindsley 1964, Haggerty 1991). Through this process, the solid solution of magnetite and ulvospinel is transformed into magnetite and $\mathrm{T}$ Ilm intergrowths $(\mathrm{Mt}+\mathrm{T} \mathrm{Ilm})$ that replace the primary titanomagnetite crystal. $\mathrm{Mt}+\mathrm{T}$ Ilm is the main kind of magnetite in Bannach Granite (Figs. 8A, 8B). It is impoverished in $\mathrm{Ti}$ when associated with thicker lamellae of T Ilm (Suppl. Tab. A4, see C3_1-5 analyse). A second type is composite magnetite ( $\mathrm{Mt}+\mathrm{C} \mathrm{Ilm}$; Fig. $8 \mathrm{D})$. $\mathrm{Mt}+\mathrm{C} \mathrm{Ilm}$ and $\mathrm{Mt}+\mathrm{T}$ Ilm do not show marked compositional differences (Suppl. Tab. A4), although, in the same sample, Mt + C Ilm shows $\mathrm{TiO}_{2}$ contents slightly higher than $\mathrm{Mt}+\mathrm{T}$ Ilm. The third variety of magnetite is not associated with ilmenite and is homogeneous (Hom Mt). It occurs associated with cummingtonite-grunerite crystals (Fig. 8F) and has a composition close to pure magnetite (Suppl. Tab. A4).
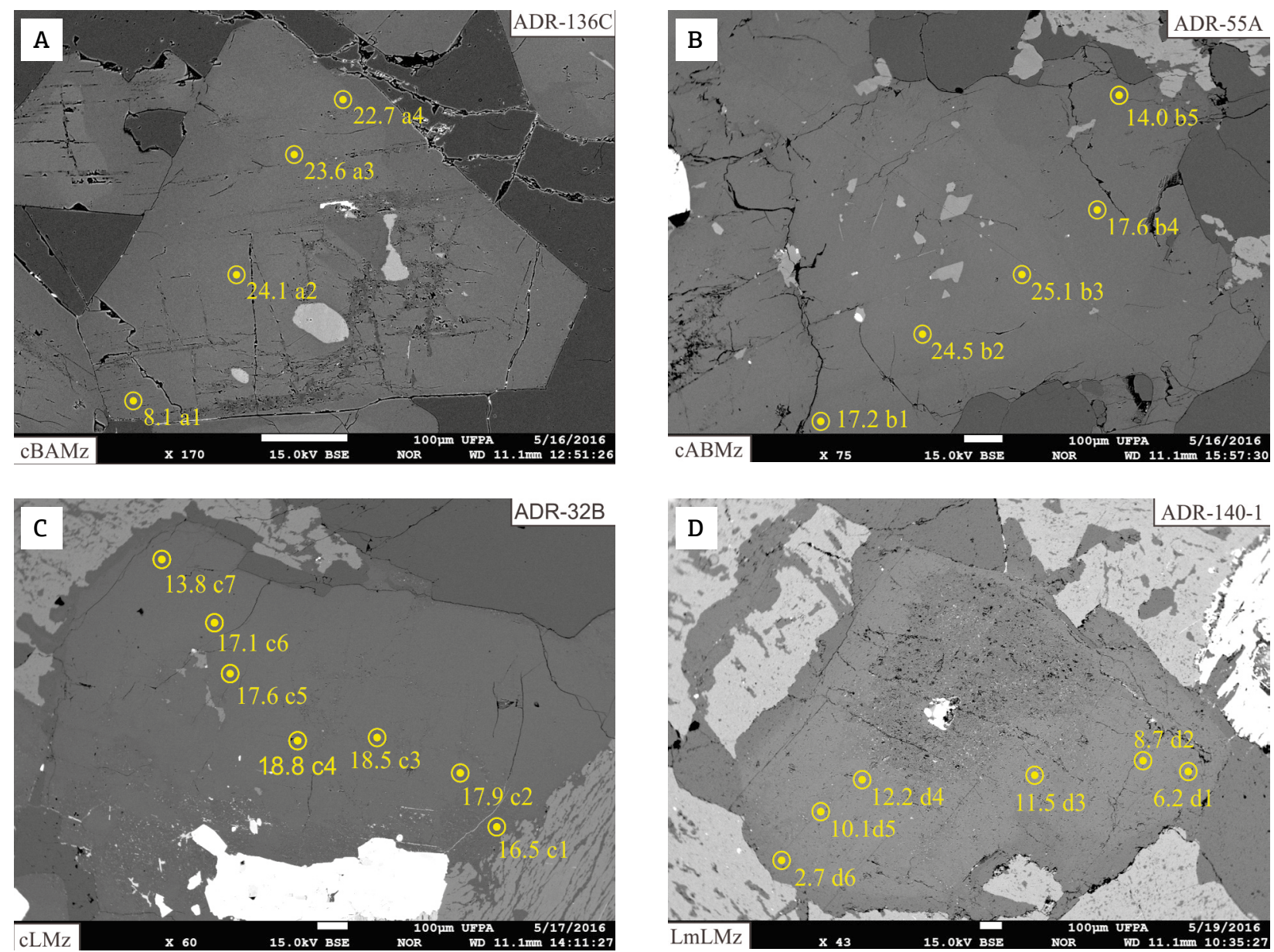

Figure 7. BSE images of subhedral to euhedral plagioclase crystals of different facies of the Bannach Granite showing the spots of microprobe analyzes (A) cBAMz facies; (B) cABMz; (C) cLMz; (D) LmLMz. The anorthite content obtained at each analyzed spot is highlighted and it is possible to observe the normal zoning of the crystals. Plagioclase compositions are giben in Table 4. 
Table 4. Representative electron microprobe analysis of plagioclase of the Bannach Granite (continued).

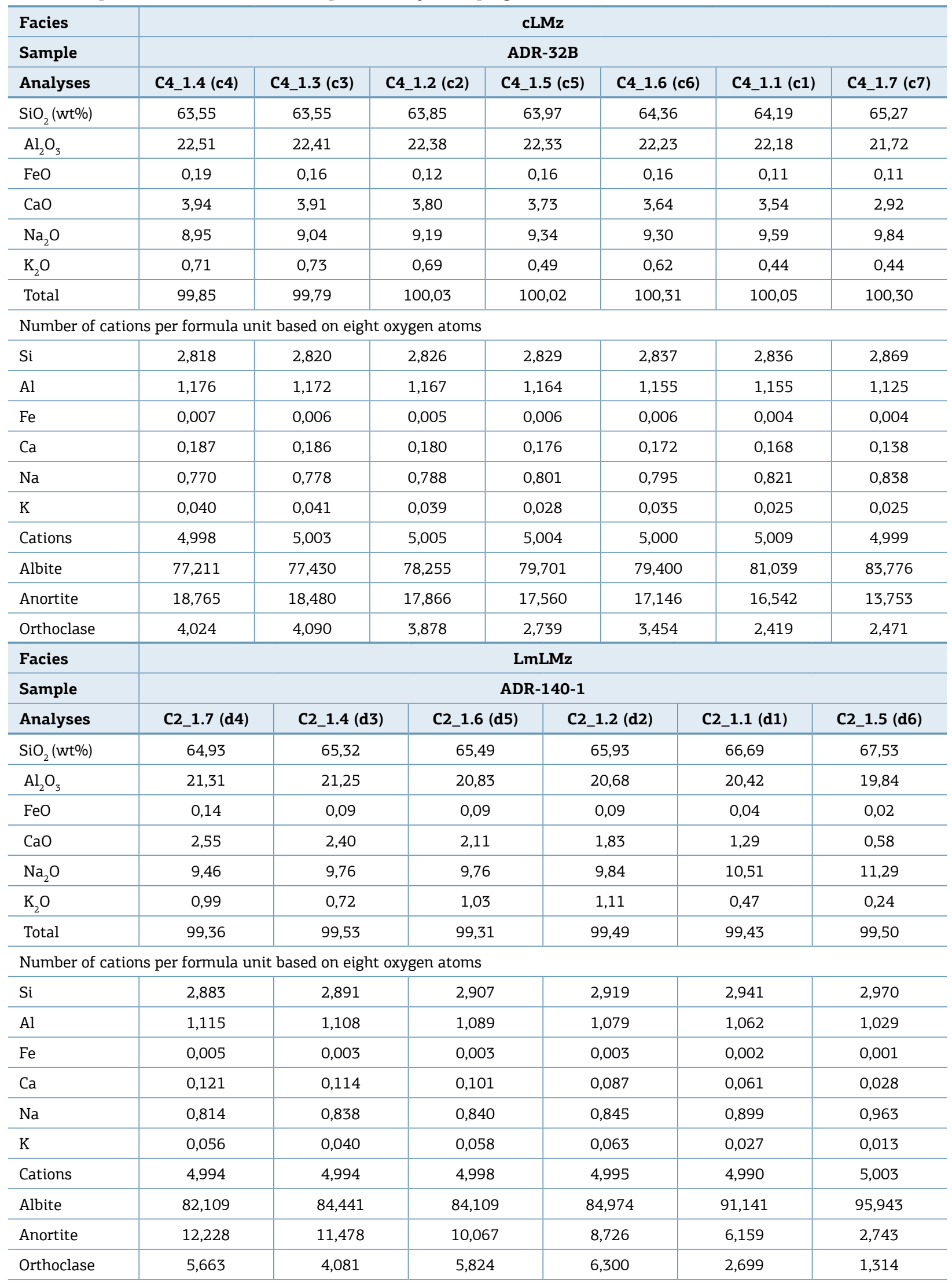

cLMz: coarse-grained leucomonzogranite; LmLMz: late medium-even grained leucomonzogranite. 

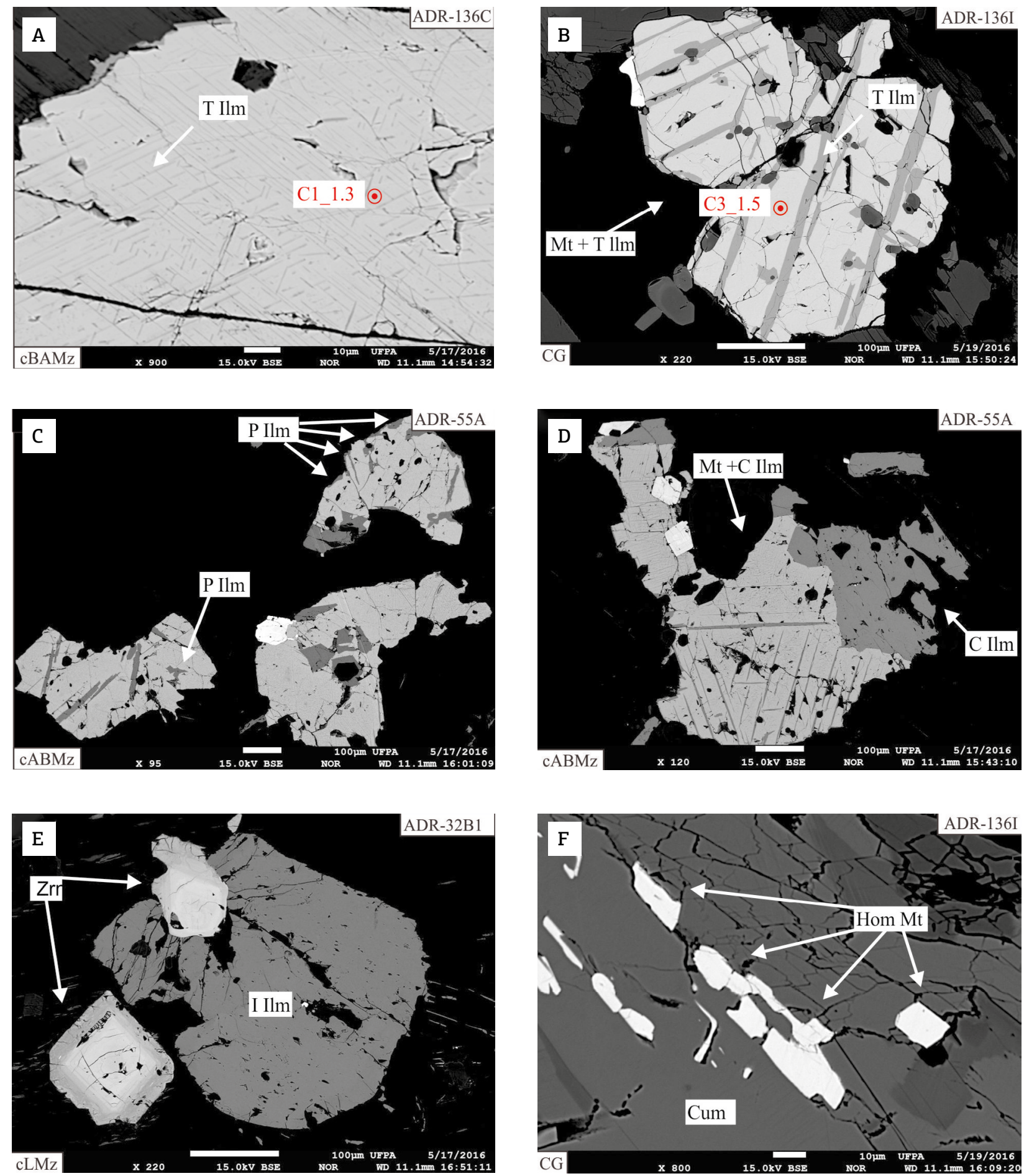

$\mathrm{T} \mathrm{Ilm}=$ trellis ilmenite; P Ilm = patch ilmenite; C Ilm = composite ilmenite. I Ilm = individual ilmenite; Mt = magnetite; Hom Mt = homogeneous magnetite; $\mathrm{Zrn}=$ zircon; Cum = cummingtonite.

Figure 8. BSE images of Fe-Ti oxide minerals of the main facies of the Bannach Granite. (A) Thin trellis ilmenite (T Ilm) lamellae in the beginning of oxi-exsolution process; (B) thick I Ilm formed in more advanced stages of the oxy-exsolution process of the magnetite forming intergrowths with trellis ilmenite (Mt $+\mathrm{T}$ Ilm) is shown in a and b images; (C) patch ilmenite (P Ilm); (D) Composite ilmenite (C Ilm) associated with magnetite (Mt + C Ilm); (E) euhedral crystal of individual ilmenite (I Ilm) associated with early crystallized zircon; (F) secondary homogeneous magnetite ( $\mathrm{H} \mathrm{Mt}$ ) associated with cummingtonite-grunerite. 


\section{DISCUSSION}

\section{Estimation of crystallization parameters of the Bannach Granite}

\section{Temperature}

Watson and Harrison (1983) developed an experimental study to evaluate the zircon saturation behavior in intermediate to felsic anatetic crustal melts and created a formula using the temperature $(\mathrm{T})$ and melt composition $(\mathrm{M})$ to estimate the required solubility of $\mathrm{Zr}$ in the magma for the zircon saturation (Eq. 1):

$\ln \mathrm{D}_{\mathrm{Zr}}=(-3.80-[0.85(\mathrm{M}-1)])+12,900 / \mathrm{T}$

Where:

$\mathrm{D}_{\mathrm{Zr}}=$ coefficient of $\mathrm{Zr}$ distribution between zircon and melt; $\mathrm{M}=(\mathrm{Na}+\mathrm{K}+(2 \mathrm{Ca}) /(\mathrm{Al} \cdot \mathrm{Si})$;

$\mathrm{T}=$ temperature in Kelvin degrees.

After, Miller et al. (2003) presented the formula proposed by Watson and Harrison (1983) as follows (Eq. 2):

$T_{\mathrm{Zr}}=12,900 /\left[2.95+0.85 \mathrm{M}+\ln \left(496,000 / \mathrm{Zr}_{\text {melt }}\right)\right]$

Where:

$\mathrm{Zr}_{\text {melt }}=$ the concentration (ppm) of $\mathrm{Zr}$ in whole rock.

Recently, Boehnke et al. (2013), using modern experimental techniques, re-evaluated the experimental data obtained by Watson and Harrison (1983) and extended the observations to pressures reaching $25 \mathrm{kbar}$. The authors concluded that their new model was not significantly different from the original of Watson and Harrison (1983), but could be applicable in pressures up to $25 \mathrm{kbar}$ and in temperatures decreasing up to $700^{\circ} \mathrm{C}$.

The proposed geothermometer equations were applied for the calculation of crystallization temperatures using whole-rock compositions of the Bannach Granite (Almeida et al. 2006). The maximum and minimum temperatures obtained for each facies are recorded in Tab. 5. This geothermometer was not applied for the cumulate facies (CG) because its composition does not correspond to a possible magmatic liquid. The zircon saturation thermometer provided relatively high temperatures ranging from 943 to $845^{\circ} \mathrm{C}$ (Tab. 5) for the cBAMz, cABMz, and $\mathrm{pBMz}$ facies and comparatively lower values for the leucogranites $\left(857\right.$ to $\left.795^{\circ} \mathrm{C}\right)$. Zircon is an early crystallized mineral in the different facies of Bannach Granite. Therefore, the results obtained are indicative of near-liquidus temperatures for the different facies.
Blundy \& Holland (1990) proposed a geothermometer by using the pair plagioclase and calcic amphibole. The experiments, done in natural and synthetic amphiboles, indicated that the $\mathrm{Al}^{\mathrm{IV}}$ content of the amphibole is strongly influenced by temperature. Their geothermometer would be suitable for amphiboles with up to $7.8 \mathrm{Si} \mathrm{pfu}$ and plagioclase with anorthite content less than $\mathrm{An}_{92}$. Thus, the composition of Bannach Granite is adequate for application of the geothermometer of Blundy and Holland (1990), according to the Equation 3:

$\mathrm{T}=(0.677 \mathrm{P}-48.98+\mathrm{Y}) /\left(-0.0429-0.008314 \ln \mathrm{K}_{1}\right)$

Where:

$\mathrm{K}_{1}=[(\mathrm{Si}-4) /(8-\mathrm{Si})] \cdot \mathrm{X}_{\mathrm{Ab}}^{\mathrm{Plag}}$;

$\mathrm{Y}=0$, for albite content $>0.5$.

A pressure value (P) of $3 \mathrm{kbar}$, obtained with the geobarometer of Mutch et al. (2016) — see "Pressure and depth of emplacement" item — was used for temperature calculations. For the amphibole-biotite bearing facies the calculated temperatures range from 807 to $733^{\circ} \mathrm{C}$, whereas in the cLMz facies, which presents a very low modal proportion of amphibole, they vary from 740 to $733^{\circ} \mathrm{C}$ (Tab. 5). It should be considered that the temperatures given by this geothermometer are indicative of near-solidus temperatures and the estimated error is $\pm 75^{\circ} \mathrm{C}$ (Blundy \& Holland 1990).

Ridolfi et al. (2010) and Ridolfi and Rezulli (2012) proposed geothermometers calibrated from the concentration of main oxides of amphiboles, resulting an error of $\geq 50^{\circ} \mathrm{C}$. Their models are applicable to amphibole-bearing granites derived from calc-alkaline to alkaline magmas with high or low water content formed under moderately reduced to moderately oxidized conditions. To estimate the crystallization temperature of Bannach Granite, the geothermometer proposed by Ridolfi et al. (2010) was employed. Moreover, the geothermometer of Putirka (2016; [Eq. (4)]) using calibrated natural and experimental calcic amphibole data from Ridolfi et al. (2010), with an estimated error of $\pm 30^{\circ} \mathrm{C}$, was also applied (Tab. 5).

$\mathrm{T}\left({ }^{\circ} \mathrm{C}\right)=1201.4-97.93\left(\mathrm{Si}^{\mathrm{Amp}}\right)+201.82\left(\mathrm{Ti}^{\mathrm{Amp}}\right)+$ $72.85\left(\mathrm{Mg}^{\text {Amp }}\right)+88.9\left(\mathrm{Na}^{\text {Amp }}\right)+40.65[\mathrm{P}(\mathrm{GPa})$.

The obtained temperatures for the $\mathrm{cBAMz}$ and $\mathrm{cABMz}$ facies by the different geothermometers show large variations. Tab. 5 displays the temperature values obtained for these facies by using the different methods. The maximum values are given by the zircon geothermometer. Compared to the zircon temperatures, those given by the geothermometer of Ridolfi et al. (2010) show a moderate decrease and 
those resulting from Putirka (2016) and Blundy and Holland (1990) geothermometers an accentuate decrease. Besides, in the cLMz facies, the temperatures obtained by the geothermometer of Blundy and Holland (1990) are lower than those calculated by other methods. This is probably due to the markedly more sodic composition of the plagioclase.

\section{Pressure and depth of emplacement}

The classical empirical Al-in-amphibole geobarometer of Hammarstron and Zen (1986) and Hollister et al. (1987) is based on the principle that the $\mathrm{Al}_{\mathrm{t}}$ content in amphibole has a positive relationship with the pressure. It is applicable to intermediate to felsic rocks in which an equilibrium of eight mineral phases plus residual liquid and volatile at the end of their crystallization occurs. In turn, Johnson and Rutherford (1989) formulated a geobarometer based on experiments developed in presence of a fluid phase formed by mixed $\mathrm{H}_{2} \mathrm{O}-\mathrm{CO}_{2}$. Blundy and Holland (1990) also carried out experimental studies and concluded that the concentration of $\mathrm{Al}^{\mathrm{IV}}$ in amphiboles is more sensitive to

Table 5. Estimation of temperature and pressure for the different facies of the Bannach Granite.

\begin{tabular}{|c|c|c|c|c|c|c|c|c|c|c|c|c|}
\hline \multirow{2}{*}{$\begin{array}{l}\text { Facies } \\
\text { Temperature }\left({ }^{\circ} \mathrm{C}\right)\end{array}$} & \multicolumn{2}{|c|}{ cBAMz } & \multicolumn{2}{|c|}{ cABMz } & \multirow{2}{*}{$\begin{array}{c}\text { pBMz } \\
\text { Max }\end{array}$} & \multicolumn{2}{|c|}{ cLMz } & \multirow{2}{*}{$\begin{array}{c}\text { EmLMz } \\
\text { Max }\end{array}$} & \multicolumn{2}{|c|}{ LmLMz } & \multicolumn{2}{|c|}{ fLMz } \\
\hline & Max & Min & $\operatorname{Max}$ & Min & & $\operatorname{Max}$ & Min & & $\operatorname{Max}$ & Min & Max & Min \\
\hline Zircon (Watson \& Harrison 1983) & 943 & 910 & 906 & 845 & 894 & 838 & 814 & 815 & 857 & 820 & 812 & 795 \\
\hline $\begin{array}{l}\text { Amphibole and plagioclase } \\
\text { (Blundy \& Holland 1990) }\end{array}$ & 786 & 733 & 807 & 733 & - & 741 & 733 & - & - & - & - & - \\
\hline Amphibole (Ridolfi et al. 2010) & 866 & 837 & 863 & 820 & - & 859 & 850 & - & - & - & - & - \\
\hline Amphibole (Putirka 2016) & 760 & 730 & 758 & 715 & & 781 & 741 & & & & & \\
\hline \multicolumn{13}{|l|}{$\begin{array}{l}\text { Pressure (kbar) } \\
\text { Al-in-amphibole geobarometer }\end{array}$} \\
\hline Hammarstron \& Zen (1986) & 4,12 & 2,98 & 4,68 & 2,97 & - & 3,22 & 2,99 & - & - & - & - & - \\
\hline Hollister et al. (1987) & 4,26 & 2,98 & 4,89 & 2,96 & - & 3,25 & 2,99 & - & - & - & - & - \\
\hline Johnson \& Rutherford (1989) & 3,30 & 2,34 & 3,77 & 2,33 & - & 2,55 & 2,35 & - & - & - & - & - \\
\hline Blundy \& Holland (1990) & 4,51 & 3,37 & 4,46 & 3,36 & - & 3,61 & 3,38 & - & - & - & - & - \\
\hline Schmidt et al. (1992) & 4,60 & 3,52 & 4,55 & 3,51 & - & 3,75 & 3,53 & - & - & - & - & - \\
\hline Mutch et al. (2016) & 3,57 & 2,83 & 3,98 & 2,82 & & 2,98 & 2,83 & - & - & - & - & - \\
\hline
\end{tabular}

Equations

Temperature $\left({ }^{\circ} \mathrm{C}\right)$

Watson \& Harrison (1983): $\mathrm{T}_{\mathrm{Zr}}=12,900 /\left[2.95+0.85 \mathrm{M}+\ln \left(496,000 / \mathrm{Zr}_{\text {melt }}\right)\right]$;

Blundy $\odot$ Holland $(1990): T\left( \pm 75^{\circ} \mathrm{C}\right)=(0.677 \mathrm{P}-48.98+\mathrm{Y}) /(-0.0429-0.008314 \ln \mathrm{K} 1)$;

Ridolfi et al. (2010): $\mathrm{T}\left(\geq 50^{\circ} \mathrm{C}\right)=151.487 \mathrm{Si}^{*}+2014 ; \mathrm{Si}^{*}=\mathrm{Si}+\left(\mathrm{Al}^{\mathrm{IV}} / 15\right)-\left(2 \mathrm{Ti}^{\mathrm{IV}}\right)-\left(\mathrm{Al}^{\mathrm{VI}} / 2\right)-\left(\mathrm{Ti}^{\mathrm{VI}} / 1.8\right)+\left(\mathrm{Fe}^{3+} / 9\right)+\left(\mathrm{Fe}^{2+} / 3.3\right)+$ $(\mathrm{Mg} / 26)+\left(\mathrm{Ca}^{\mathrm{B}} / 5\right)+\left(\mathrm{Na}^{\mathrm{B}} / 1.3\right)-\left(\mathrm{Na}^{\mathrm{A}} / 15\right)+\left([]^{\mathrm{A}} / 2.3\right)$

Putirka (2016): $\mathrm{T}\left( \pm 30^{\circ} \mathrm{C}\right)=1201.4-97.93\left(\mathrm{Si}^{\mathrm{Amp}}\right)+201.82\left(\mathrm{Ti}^{\mathrm{Amp}}\right)+72.85\left(\mathrm{Mg}^{\mathrm{Amp}}\right)+88.9\left(\mathrm{Na}{ }^{\mathrm{Amp}}\right)+40.65[\mathrm{P}(\mathrm{GPa})]$

Pressure (kbar)

Hammarstron $\odot$ Zen (1986): $\mathrm{P}( \pm 3 \mathrm{kbar})=-3.92+5.03 \mathrm{Al}_{\mathrm{t}} ;$ Hollister et al. (1987): $\mathrm{P}( \pm 1 \mathrm{kbar})=-4.76+5.64 \mathrm{Al}$

Johnson $\odot$ Rutherford (1989): P $( \pm 0.5 \mathrm{kbar})=4.23$ Alt - 3.46; Blundy $\odot$ Holland (1990): P $(\mathrm{kbar})=5.03 \mathrm{Al}$ - 3.53 ;

Schmidt (1992): P $( \pm 0.6 \mathrm{kbar})=4.76 \mathrm{Alt}-3.01$;

Mutch et al. (2016): $\mathrm{P}( \pm 0.5 \mathrm{kbar})=0.5+0.331(8) * \mathrm{Al}_{\mathrm{t}}+0.995(4) *\left(\mathrm{Al}_{\mathrm{t}}\right)^{2}$

Note: Only analyzes in crystal rim were used for amphibole pressure and temperature calculations; CG: even-grained cumulatic granite; cBAMz: even coarsegrained biotite-amphibole monzogranite; cABMz: even coarse-grained amphibole-biotite monzogranite; pBMz: porphyritic biotite monzogranite; cLMz: even coarse-grained leucomonzogranite; EmLMz: early even medium-grained leucomonzogranite; LmLMz: late even medium-grained leucomonzogranite; fLMz: even fine-grained leucomonzogranite. 
temperature than to pressure. Schmidt et al. (1992) developed a geobarometer from experimental calibration of the assemblage amphibole + biotite + plagioclase + orthoclase + quartz + titanite, and Fe-Ti oxides + residual liquid + volatiles. Anderson and Smith (1995) stated that pressure calculation is affected by temperature and concluded that the $\mathrm{Al}$-in-amphibole geobarometer was not suitable for high $\mathrm{Fe} /(\mathrm{Fe}+\mathrm{Mg})$ rocks formed under reduced conditions, and for amphiboles with Fe\# $>0.65$.

More recently, new geobarometers based on the $\mathrm{Al}$ content of calcic amphibole were proposed (Ridolfi et al. 2010, Ridolfi \& Rezulli 2012). However, Erdmann et al. (2014) concluded that the geobarometers provided by these authors would reflect more compositional variations of the magma than pressure effects. Putirka (2016) also made restrictions to the use of $\mathrm{Al}$-in-amphibole geobarometers, except in those cases where the constraints indicated by Anderson and Smith (1995) are taken into account.

Finally, Mutch et al. (2016) developed a geobarometer integrating selected experimental data from the literature with a wide range of new experimental data. Their calibration was performed at near-solidus temperatures and over a large range of pressures from $0.8 \mathrm{kbar}$, close to the minimum pressure of amphibole stability, down to $10 \mathrm{kbar}$. This geobarometer is applicable in a large variety of granitoid rocks containing the mineral assemblage amphibole + plagioclase $\left(A n_{15-80}\right)+$ biotite + alkali feldspar + quartz + magnetite + ilmenite/titanite + apatite. Such mineral association is found in the Bannach Granite, and thus we can use this geobarometer to unravel its pressures of emplacement.

To go far in the evaluation of the applicability of that geobarometer for rocks of the Bannach Granite, the compositions of the amphiboles studied here are compared with those of Mutch et al. (2016). Despite a slightly higher content of $\mathrm{Al}^{\mathrm{IV}}$ in Bannach Granite, the diagrams show that the compositions of amphibole from cBAMz, cABMz, and cLMz facies are similar to those of Mutch et al. (2016; experimental and natural material), and mainly for the sample of granodiorite FSC1332 (Figs. 9A, 9B, 9C). Additionally, as noted by Mutch et al. (2016; Tab. 2), the rocks selected by them for experimental studies present lower $\mathrm{Al}_{2} \mathrm{O}_{3}$ content than the typical Cordilleran calc-alkaline associations, and this is also observed in the Bannach and Jamon Suite granites (Dall'Agnol et al. 1999b, 1999c; 2005).

The equation proposed by Mutch et al. (2016) to calculate the emplacement pressure is (Eq. 5):

$\mathrm{P}(\mathrm{kbar})=0.5+0.331(8) * \mathrm{Al}_{\mathrm{t}}+0.995(4) *\left(\mathrm{Al}_{\mathrm{t}}\right)^{2}$

The results obtained by the applied geobarometers show significant differences (Tab. 5). The geobarometers of Blundy and Holland (1990), Schmidt (1992), Hammarstron and Zen (1986),
A

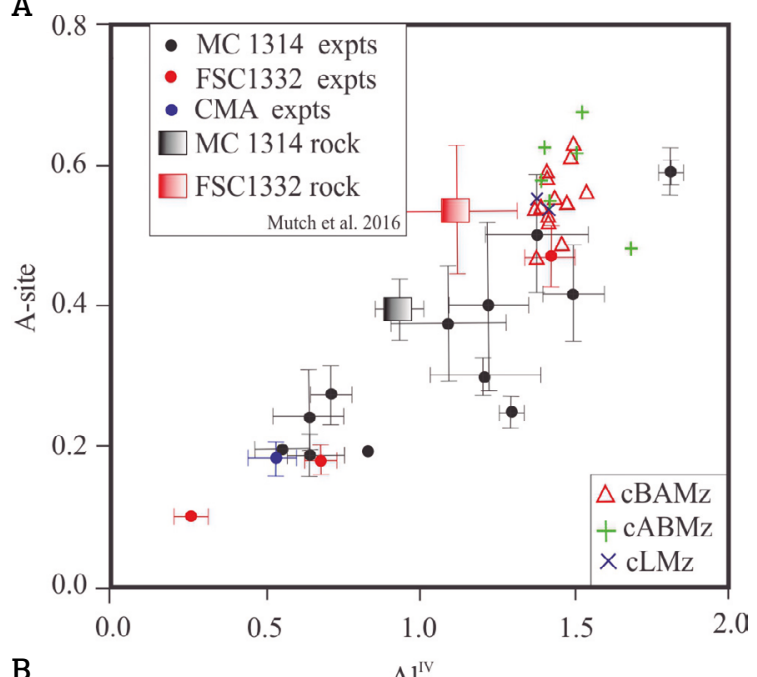

B
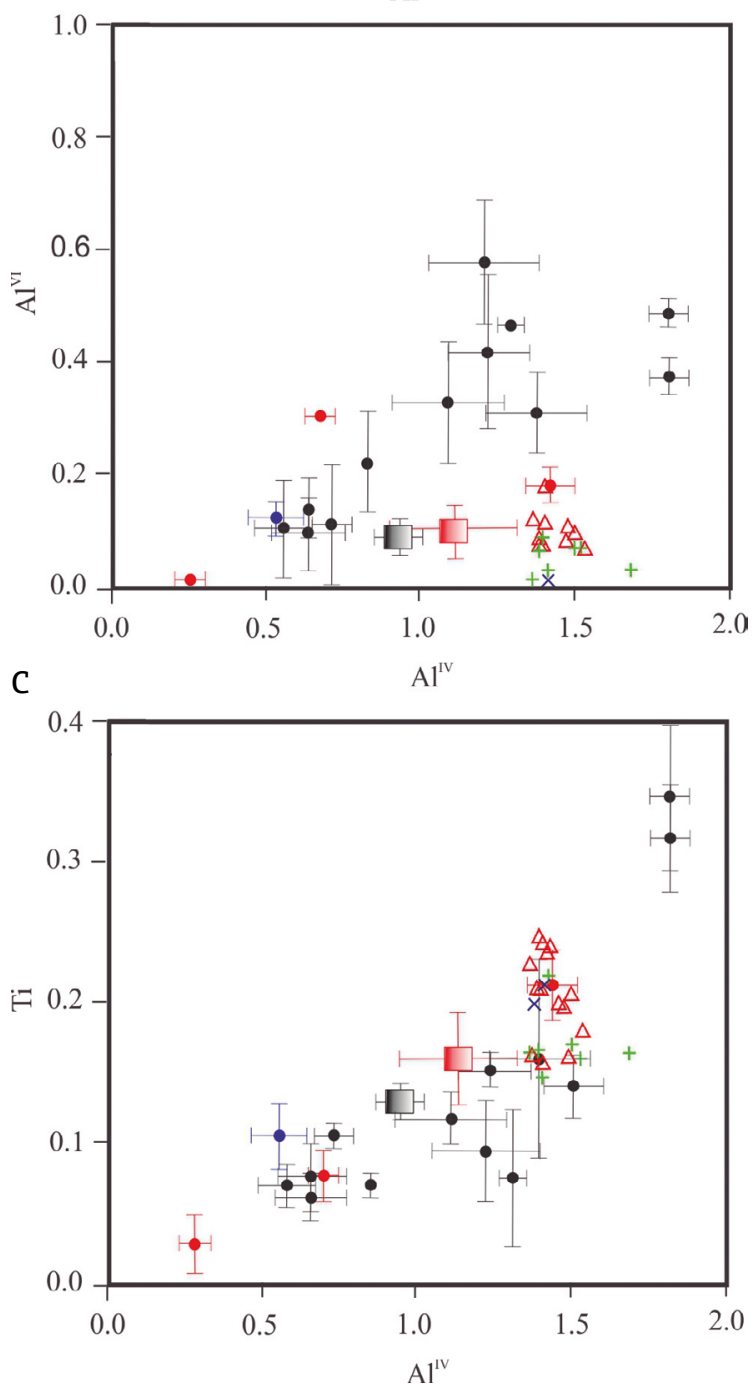

Figure 9. Comparison between the compositional variation of the experimental (MC 1314, FSC 1312 and CMA) and natural amphibole (MC 1314 rock and FSC 1332 rock) of Mutch et al. (2016) and calcic amphiboles of the Bannach granite. (A) $\mathrm{Al}^{\mathrm{IV}} \times \mathrm{A}$-site occupancy ( $\mathrm{Na}$ $+\mathrm{K})$; (B) $\mathrm{Al}^{\mathrm{IV}} \times \mathrm{Al}^{\mathrm{VI}}$; (C) $\mathrm{Al}^{\mathrm{IV}} \times \mathrm{Ti}$. 
and Hollister et al. (1987) provided averaged pressures of ca $4 \pm 0.5 \mathrm{kbar}$, whereas those of Johnson and Rutherford (1989) and Mutch et al. (2016) indicate pressures around $3 \pm 0.5 \mathrm{kbar}$.

Aiming to estimate the pressure, amphibole compositions of Bannach Granite were plotted in the diagram P (kbar) $\mathrm{Al}^{\text {tot }}$ (Fig. 10; based on Mutch et al. 2016 and their Fig. 10C). In this figure, the experimental data of Johnson and Rutherford (1989), Schmidt (1992), and Mutch et al. (2016) are also shown. The compositions of Bannach amphibole are more compatible with the experimental data of Mutch et al. (2016) and Johnson and Rutherford (1989), diverging to some extent from those of Schmidt (1992).

Given that the various facies of Bannach are exposed at the same topographic level and there is no evidence of tectonic displacement (Oliveira et al. 2008), we conclude that a emplacement pressure of about $3 \pm 0.5 \mathrm{kbar}$ can be assumed for the granite. This value is compatible with the geological setting of the Bannach Granite and it is coincident with that estimated for the Jamon Granite (Dall' Agnol et al. 1999c).

In extensional tectonic settings, magmatic intrusions are generally laccoliths, as exemplified by the classical rapakivi granite plutons (Vigneresse 2005). Geophysical gravity survey indicated that the Bannach Granite is $2.2 \mathrm{~km}$ thick (Oliveira et al. 2008). On the other hand, the pressures obtained indicate an emplacement depth around $11.1 \pm 1.9 \mathrm{~km}$ for the pluton and an erosion of approximately $10 \mathrm{~km}$ of crust can

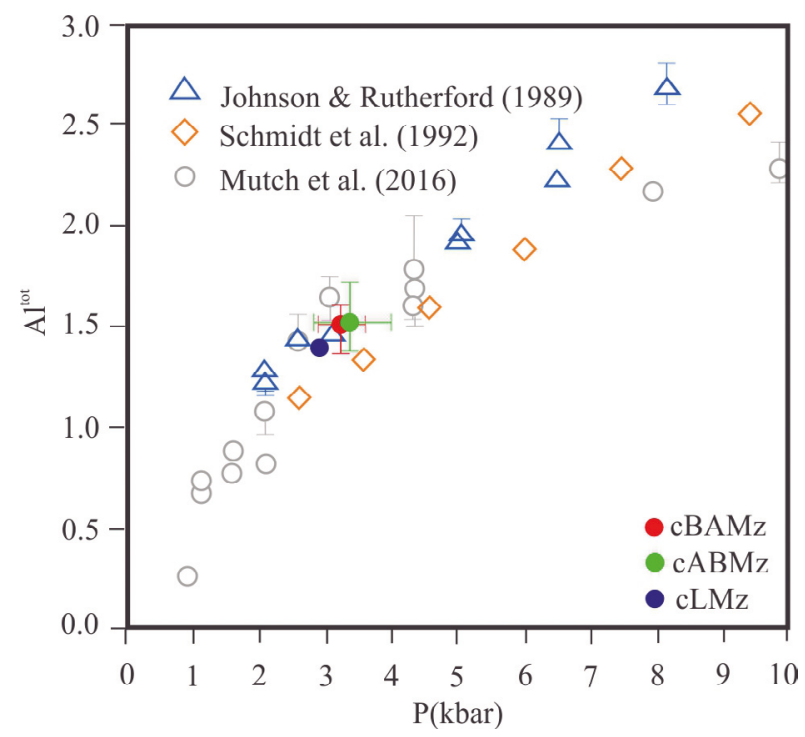

Figure 10. $\mathrm{P}(\mathrm{kbar}) \times \mathrm{Al}^{\text {tot }}$ diagram showing the compositional variation of rims of calcic amphibole of the different facies of the Bannach Granite. Calcic amphibole compositions of Johnson \& Rutherford (1989), Schmidt (1992) and Mutch et al. (2016) are also plotted for comparison. The diagram indicates $3 \pm 0.5$ kbar pressures (based on Mutch et al. 2016, Fig. 10C) for the Bannach Granite emplacement. be estimated (until the exhumation of the pluton). Moreover, accepting the model proposed for the Jamon Suite, in which the magmas were generated at the base of the continental crust by partial melting caused by underplating of mafic magmas (Dall'Agnol et al. 2005; Oliveira et al. 2008, 2010a), it can be concluded that the estimated emplacement depth was different from the depth of Bannach magma generation.

\section{Oxygen fugacity $\left(\mathrm{fO}_{2}\right)$}

Almeida et al. (2006) estimated that the Bannach Granite would have formed under oxygen fugacity conditions similar the other oxidized A-type granites of the Jamon Suite from Carajás Province (Dall'Agnol et al. 2005). Amphibole and biotite compositions and the assemblage of Fe-Ti oxide minerals have been commonly utilized to estimate oxygen fugacity during magmatic evolution because they are strongly dependent of $\mathrm{fO}_{2}$ (Carmichael 1966, Ishihara 1981, Wones 1989, Carmichael 1991, Frost \& Lindsley 1991, Frost 1991, Clark 1999, Dall'Agnol et al. 1997, 1999b, 2005, Dall'Agnol \& Oliveira 2007).

In the $\mathrm{Fe} /(\mathrm{Fe}+\mathrm{Mg})$ vs. $\mathrm{Al}^{\mathrm{IV}}$ diagram, calcic amphiboles of Bannach Granite plot in the field of intermediate $f \mathrm{O}_{2}$ except for some samples of CG facies which plot in the high fugacity field (Fig. 11A). In the $\mathrm{Al}^{\mathrm{IV}}+\mathrm{Al}^{\mathrm{VI}}$ vs. $\mathrm{Fe} /(\mathrm{Fe}+\mathrm{Mg})$ plot, the biotite of CG, cBAMz, cABMz, and cLMz facies shows Fe/ $(\mathrm{Fe}+\mathrm{Mg})$ ratios similar to those observed in the Proterozoic anorogenic magnetite series granites of Laurentia (Fig. 11B; fields of Anderson \& Bender 1989; biotite variations in the ilmenite and magnetite series, as suggested by Anderson et al. 2008). Additionally, that diagram suggests oxygen fugacity conditions between FMQ and FMQ + 1 for the Bannach pluton, corresponding to low oxidizing conditions. However, the constant presence of magnetite associated with magmatic titanite and the amphibole compositions (Fig. 11A) are indicative of crystallization under more oxidizing conditions (Frost \& Lindsley 1991, Wones 1989, Dall' Agnol et al. 1999c, Frost et al. 2000). Dall'Agnol et al. (1997, 1999c) estimated fugacity conditions a little higher than the NNO buffer (NNO +0.5 ) for the Jamon Granite crystallization. The Jamon and Bannach granites show petrographic and mineralogical analogies, particularly regarding the Fe-Ti oxides assemblage and amphibole composition (Fig. 11A). Nevertheless, the biotites of the cBAMzc, cABMzc, and cLMz facies, have a slightly higher $\mathrm{Fe} /(\mathrm{Fe}+\mathrm{Mg})$ ratio than those of the Jamon Granite (Fig. 11B). It suggests that Bannach Granite may have crystallized under a little lower oxygen fugacity (NNO to NNO0.5) compared to that admitted for Jamon Granite.

A different behavior is observed in the LmLMz facies of Bannach Granite, which despite having magnetite exhibits very high $\mathrm{Fe} /(\mathrm{Fe}+\mathrm{Mg})$ ratios $(0.89$ to 0.91$)$ in biotite. As a result, it plots in the field of the ilmenite series (Fig. 11B) and reveals analogies with granites formed under reduced 
conditions (Anderson \& Smith 1995, Anderson et al. 2008, Dall'Agnol et al. 2005, Cunha et al. 2016). Almeida et al. (2006) had highlighted that the LmLMz facies differs from other Bannach facies, not only in field occurrence but also in
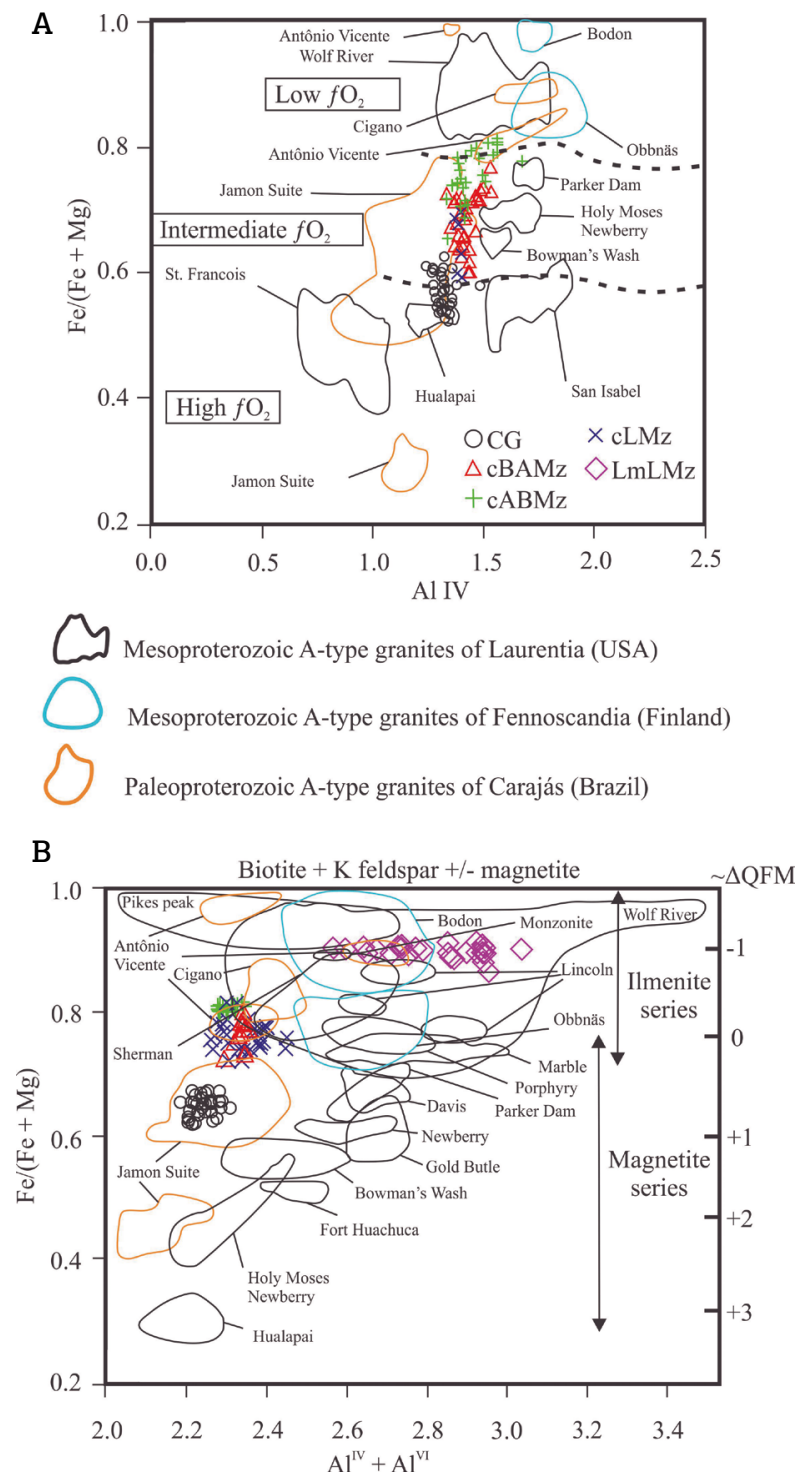

Figure 11. Comparison of amphibole and biotite composition of the Bannach Granite with similar granites from Carajás Province, Laurentia and Fennoscandia (based on Dall'Agnol et al. 2005). (A) $\mathrm{Al}^{\mathrm{IV}}$ vs. $\mathrm{Fe} /(\mathrm{Fe}+\mathrm{Mg})$ diagram showing the amphibole compositions of the different facies of the Bannach Granite; (B) $\mathrm{Al}^{\mathrm{IV}}+\mathrm{Al}^{\mathrm{VI}}$ vs. $\mathrm{Fe} /(\mathrm{Fe}+\mathrm{Mg})$ diagram showing the biotite compositions of the different facies of the Bannach Granite. Ilmenite and magnetite series variation and $\triangle \mathrm{QFM}$ according to Anderson et al. (2008). Amphiboles and biotites of the A-type Paleoproterozoic granites from Carajás Province (Dall'Agnol et al. 2005) and Mesoproterozoic Granites of the Laurentia (Anderson \& Smith 1995, Frost et al. 1999) and Fennoscandia (Rämö \& Haapala 1995, Elliott 2001, Kosunen 2004) are plotted for comparison. the geochemical behavior of $Y$ and rare earth elements (REE). Furthermore, that facies does not show a clear relation with other facies in terms of magmatic differentiation. Considering these evidences, they concluded that the LmLMz facies should be a product of independent magmatic pulses. The new mineralogical data presented in this work reinforce this hypothesis since they indicate that the LmLMz was derived from a more reduced source compared to that of other Bannach facies.

\section{Water content}

Like pressure, temperature and oxygen fugacity, the water content has a direct influence in mineral stability. The amphibole, for example, is extremely dependent of this parameter (Dall' Agnol et al. 1999c, Klimm et al. 2003). Oliveira et al. (2010c) studied a granodiorite with high modal amphibole content and their experiments at $\mathrm{T}$ between $950-680^{\circ} \mathrm{C}$ estimated a minimum water content in the magma of $7 \mathrm{wt} . \%$. In a similar way, experiments carried out at $300 \mathrm{MPa}$ and oxidizing conditions in the Jamon Granite showed that a minimum content of -4.5 up to $-6.5 \mathrm{wt} . \%$ of water was necessary to assure magnetite + hornblende + plagioclase stability during its initial crystallization stage (Dall'Agnol et al. 1999c). Cunha et al. (2016), based on the stability of amphibole, estimated a minimum content of $4 \mathrm{wt}$ \% of water in the Planalto Granite magma. Thus, by analogy with Jamon Granite, a magma water content between 4.5 and 6.5 wt. $\%$ can also be considered for Bannach Granite, once mineral association, crystallization history, and emplacement pressure are similar in both granites.

\section{Comparisons with other A-type Paleoproterozoic granites of Carajás Province}

We have selected A-type Paleoproterozoic granites of the three suites from Carajás Province for comparisons (Dall'Agnol et al. 1999c, Dall'Agnol et al. 2005). As discussed before, Bannach Granite is akin to the granites of Jamon Suite (Dall'Agnol et al. 2005). In the less evolved facies of the Bannach Granite and the Jamon Suite, ferroan biotite and amphibole are the main mafic minerals and titanite and magnetite are primary accessory minerals.

The amphiboles of Jamon Suite are ferro-edenite to edenite (Dall'Agnol et al. 2005) while in Bannach Granite, they are ferro-edenite to ferro-hornblende and, rarely, hastingsite. The $\mathrm{Fe} /(\mathrm{Fe}+\mathrm{Mg})$ ratios of calcic amphibole of Jamon pluton range from 0.47 to 0.65 in the hornblende-biotite monzogranites and between 0.60 to 0.73 in the (hornblende)-biotite monzogranites. These ratios are similar to those obtained in calcic amphiboles from Bannach (Fig. 11A). The amphiboles of Serra dos Carajás and Velho Guilherme Suite are hastingsite with $\mathrm{Fe} /(\mathrm{Fe}+\mathrm{Mg})$ ratios ranging, respectively, from 0.85 to 0.94 and 0.74 to 0.85 higher than those of the 
studied granite (Fig. 11A). Ferromagnesian amphiboles are product of alteration of clinopyroxene and were described in several plutons of the Jamon Suite (Gastal 1987, Dall'Agnol 1982). They have also been described in Bannach Granite and classified as cummingtonite and grunerite.

Estimated temperatures for Bannach Granite (Tab. 5) are consistent with those for the hornblende-biotite monzogranite facies of Jamon Granite, in the range of 870 to $-700^{\circ} \mathrm{C}$ (Dall'Agnol et al. 2005). Otherwise, the hornblende-biotite monzogranite facies of the Serra dos Carajás and Velho Guilherme suites indicate liquidus temperature $\left(-850^{\circ} \mathrm{C}\right)$ lower than that of Jamon Suite and are distinguished from the latter by the absence of clinopyroxene and occurrence of less calcic plagioclase (Dall'Agnol et al. 2005).

$\mathrm{Al}$-in-amphibole geobarometers indicate emplacement pressures similar for Bannach and Jamon plutons. It was not possible to use Al-in-amphibole geobarometers for the Serra dos Carajás and Velho Guilherme because they have high Fe content (Anderson \& Smith 1995). However, geological evidence suggests a lower pressure $(2 \pm 1 \mathrm{kbar}$, Serra dos Carajás; $1 \pm 0.5$ kbar, Velho Guilherme; Dall'Agnol et al. 2005) for the emplacement of these suites.

In terms of oxygen fugacity, the compositions of Bannach amphibole suggest conditions similar to those of Jamon oxidized granite, although the calcic amphiboles of Bannach Granite are slightly enriched in $\mathrm{Al}^{\mathrm{IV}}$ (Fig. 11A). The composition of Bannach biotite reveals that the CG present lower Fe/ $(\mathrm{Fe}+\mathrm{Mg})$ ratio and, therefore, are comparatively more oxidized than the cBAMzc, cABMzc and cLMzc facies, which are in the transition between the magnetite and ilmenite series (as estimated by Anderson et al. 2008). Conversely, the LmLMz facies is reduced because its biotite has high $\mathrm{Fe} /(\mathrm{Fe}+\mathrm{Mg})$ ratios (0.89 to 0.91 , see Fig. 11B), and plots in the ilmenite series domain. Besides, it has higher $\mathrm{Al}^{\text {tot }}$ contents than the other facies of the Bannach Granite and also Jamon Granite (Fig. 11B). The amphibole composition of Bannach Granite indicates that, during the crystallization of the magma, fugacity conditions overlapped with those estimated for similar facies of the Jamon Granite (NNO + 0.5; Dall'Agnol et al. 1997, 1999c), though $\mathrm{Fe} /(\mathrm{Fe}+\mathrm{Mg})$ ratios in biotite reveal $f \mathrm{O}_{2}$ slightly more reducing (NNO to NNO-0.5). Besides, the LmLMz facies was apparently generated under reduced conditions, because its biotite is similar in composition to those of the Cigano (Serra dos Carajás Suite) and Antônio Vicente (Suite Velho Guilherme) granites.

\section{Comparisons with A-type granites from other provinces of the world}

The granites selected for comparison are the Mesoproterozoic granites of the magnetite and ilmenite series of Laurentia (Anderson \& Bender 1989, Anderson \& Smith 1995, Anderson \& Morrison 2005) and the rapakivi granites of Finland (Wiborg Batholith and Obbnäs and Bodon plutons; Rämö \& Haapala 1995; Elliott 2001; Kosunen 2004). In the amphibole-bearing facies of the Bannach Granite, ilmenite is subordinate and magnetite is the main opaque mineral. Hence, they belong to the magnetite series (Ishihara 1981). In addition, titanite is a significant primary accessory in these facies. The amphibole and biotite have compositions comparable to those of the granites of the magnetite series of Laurentia (Fig. 11). There is a strong $\mathrm{Fe} /(\mathrm{Fe}+\mathrm{Mg})$ ratio similarity between the calcic amphibole and biotite of Bannach and those of the Parker Dam, Holy Moses, Newberry, Bowman's Wash, San Isabel and Hualapai granites, though, except for Hualapai, the granites mentioned have higher $\mathrm{Al}^{\mathrm{IV}}$ and $\mathrm{Al}^{\mathrm{t}}$ contents. In relation to biotite, the Holy Moses and Hualapai granites show lower $\mathrm{Fe} /(\mathrm{Fe}+\mathrm{Mg})$ ratios than Bannach Granite.

Fe-Ti oxides and diagrams displayed on Figure 11 suggest that the amphibole-bearing facies of Bannach Granite were formed under oxidizing conditions, similar to most of the magnetite series granites of Laurentia (Anderson \& Smith 1995, Anderson \& Morrison 2005). On the other hand, higher $\mathrm{Al}$ content in the amphibole can indicate the formation of Laurentia granites at higher pressures than the Jamon Suite. However, it is necessary prudence in the use of the Al-inamphibole geobarometer due to the method limitations put in evidence by Anderson and Smith (1995). They showed that the estimated pressures for the emplacement of most of the Laurentia oxidized granites were probably overestimated by $2 \pm 1$ kbar (Anderson \& Smith 1995, their Fig. 8). Taking this into account, there would be no significant difference of emplacement pressure between the granites of Jamon Suite and the oxidized Mesoproterozoic granites (Parker Dam, Holy Moses, Newberry and Bowman's Wash) of Laurentia.

Wolf River Batholith (Anderson et al. 2008) and the rapakivi granites of Finland (Rämö \& Haapala 1995, Elliott 2001, Kosunen 2004) exhibit amphiboles of similar composition ranging from ferro-edenite to hastingsite, with associated annite biotite. In addition, magnetite is rare or absent and they are classified as ilmenite series granites (Ishihara 1981), except the Obbnäs granite which is slightly more oxidized and contains magnetite. These granites were formed under low oxygen fugacity conditions close to the FMQ buffer (Fig. 11B). The estimated temperatures for the Wolf River Batholith are between 640 to $790^{\circ} \mathrm{C}$ (Anderson 1980), with a slightly lower minimum temperature than those recorded in the Bannach Granite. On the other hand, the obtained temperature by the geothermometer of Blundy and Holland (1990) for the Wiborg Batholith is $-740^{\circ} \mathrm{C}$ (Elliot 2001). Al-in-amphibole geobarometers provided emplacement pressures of $-5 \mathrm{kbar}$ for the Wolf River Batholith (Anderson \& Smith 1995) and in the range of 2.5 to $5.4 \mathrm{kbar}$ for the Wiborg Batholith (Elliot 2001). However, the high Fe 
content of their amphiboles limits the application of the Al-inamphibole geobarometer (Anderson \& Smith 1995) and such pressures appear overestimated. Emplacement pressures below $2 \mathrm{kbar}$ are more likely in the case of Wolf River (Anderson \& Smith 1995) and, based on geological evidence (see discussion in Elliot 2001), probably would also not be higher than $2 \mathrm{kbar}$ in the case of Wiborg. Therefore, these granites were formed under more reducing conditions and were probably emplaced at lower pressures than the Bannach pluton. On the other hand, considering the aforementioned mineral and chemical characteristics, the evidence of its formation under reducing conditions (Fig. 11B), the LmLMz facies of Bannach Granite approaches the ilmenite series granites of Laurentia and the rapakivi granites of Finland.

\section{CONCLUSIONS}

The Bannach Granite has calcic amphibole (ferro-hornblende and ferro-edenite, locally hastingsite) with $\mathrm{Fe} /(\mathrm{Fe}+$ $\mathrm{Mg}$ ) ratio ranging from 0.53 to 0.81 , and subordinate cummingtonite-grunerite with $\mathrm{Fe} /(\mathrm{Fe}+\mathrm{Mg})$ ratio in between 0.45 and 0.57 . The biotite has $\mathrm{Fe} /(\mathrm{Fe}+\mathrm{Mg})$ ratio $>0.60$ and this ratio increases along the trend: $\mathrm{GC} \rightarrow \mathrm{cBAMz} \rightarrow$ $\mathrm{cABMZ} \rightarrow \mathrm{cLMz} \rightarrow \mathrm{LmLMz}$ and approaches annite composition in the latter variety. The plagioclase presents normal zoning with andesine sodic or calcic oligoclase cores and sodic oligoclase rims in less evolved facies and calcic to sodic oligoclase cores with albite rims in the more evolved facies.

Titanite exhibit Fe/Al ratios between 0.48 to 0.57 , and together with textural evidence, this indicates its magmatic origin. Magnetite and ilmenite are present in all facies of the granite. The most common textural variety of ilmenite is trellis, though patches, composite, and individual ones also occur. Magnetite forms intergrowths with trellis ilmenite, associated with composite ilmenite or occurring as homogeneous crystals. There are no expressive compositional contrasts between the textural varieties of ilmenite. The magnetite is always Ti-poor, and the homogeneous crystals are associated with cummingtonite-grunerite and are practically devoid of Ti.

The magmas of the Bannach Granite were formed at high temperatures. The estimated near-liquidus temperatures vary between 943 and $795^{\circ} \mathrm{C}$ and the near-solidus temperatures between 807 and $732^{\circ} \mathrm{C}$, in both cases decreasing from the more mafic to the more felsic facies. Emplacement pressures of $3 \pm 0.5 \mathrm{kbar}(11.1 \pm 1.9 \mathrm{~km})$ are estimated for the pluton.

Based on mineralogical characteristics and analogies with the Jamon Granite, a water content of -4.5 to 6.5 wt. $\%$ was estimated in the dominant magma of Bannach Granite. The $\mathrm{Fe} /(\mathrm{Fe}+\mathrm{Mg})$ ratios of amphibole and the presence of magnetite and magmatic titanite suggest that the amphibole-bearing
Bannach granites crystallized under moderately oxidizing conditions, slightly above nickel-nickel-oxide (NNO) buffer similar to those of Jamon magmas, but biotite compositions point to slightly more reducing conditions. Hereby, we propose that the crystallization of the dominant facies in the Bannach occurred under oxygen fugacity conditions corresponding to $\mathrm{NNO}$ to NNO-0.5. Although also containing magnetite, the $\mathrm{LmLMz}$ represents an exception due to its high $\mathrm{Fe} /(\mathrm{Fe}+\mathrm{Mg})$ ratio in biotite and crystallized under lower oxygen fugacity compared to the other Bannach facies.

When compared to the A-type Paleoproterozoic granites from Carajás Province, Bannach Granite shows strong similarity with the oxidized A-type granites of Jamon Suite and presents marked differences in relation to moderately reduced or reduced granites of the Serra dos Carajás and Velho Guilherme suites, respectively. In turn, the LmLMz facies is an exception due to their $f \mathrm{O}_{2}$ analogies with the reduced granites of the province.

Among the A-type granites of other cratons selected for comparison, Bannach Granite shows higher similarity with the granites of the magnetite series of Laurentia (USA) crystallized under moderately oxidizing conditions. The Mesoproterozoic Wolf River Batholith of the ilmenite series from Laurentia (USA) and the Fennoscandian rapakivi granites differ from the dominant facies of Bannach Granite, because they were formed in reduced fugacity conditions.

\section{ACKNOWLEDGEMENTS}

The authors are thankful to: the Institute of Geosciences and the Graduate Program in Geology and Geochemistry of the Universidade Federal do Pará (UFPA) for the infrastructure made available; G.T. Marques and A.P.P. Corrêa for their assistance with, respectively, the electron microprobe and scanning electron microscope mineral chemical analyses conducted at the Laboratório de Microanálises of UFPA; E. P. Oliveira and H. T. Costi for their reviews of a preliminary version of the manuscript; M. F. B. Teixeira for the Englih version of the manuscript and I. R. V. Cunha for assistance in mineral chemistry; CNPQ for scholarships to CJSM (Proc. 132490/2015-3) and grant to RD (Proc. 306108/2014-3). This research received financial support from the INCT program (CNPq/FAPESPA/CAPES/PETROBRAS; Proc. 573733/2008-2). This paper is a contribution to the Brazilian Institute of Amazonian Geosciences (INCT GEOCIAM).

\section{SUPPLEMENTARY DATA}

Supplementary data associated with this article can be found in the online version: Suplementary Table A1-A4. 


\section{REFERENCES}

Almeida J.A.C., Dall'Agnol R., Dias S.B., Althoff F.J. 2010. Origin of the Archean leucogranodiorite-granite Suites: Evidence from the Rio Maria terrane and implications for granite magmatism in the Archean. Lithos, 120:235-257. DOI: 10.1016/j.lithos.2010.07.026

Almeida, J.A.C., Dall'Agnol R., Leite A.A.S. 2013. Geochemistry and zircon geochronology of the Archean granite Suites of the Rio Maria granite-greenstone terrane, Carajás Province, Brazil. Journal of South American Earth Sciences, 42:103-126. https://doi.org/10.1016/j. jsames.2012.10.008

Almeida J.A.C., Dall'Agnol R., Oliveira D.C. 2006. Geologia, Petrografia e Geoquímica do Granito Anorogênico Bannach, Terreno GranitoGreenstone de Rio Maria, Pará. Revista Brasileira de Geociências, 36:282-295

Almeida J.A.C., Dall'Agnol R., Oliveira M.A., Macambira M.B. Pimentel M.M., Rämö O.T., Guimarães F.V., Leite A.A.S. 2011. Zircon geochronology and origin of the TTG Suites of the Rio Maria granitegreenstone terrane: Implications for the growth of the Archean crust of the Carajás province; Brazil. Precambrian Research, 187:201221. DOI: 10.1016/j.precamres.2011.03.004

Almeida J.A.C., Guimarães F.V., Dall'Agnol R. 2007. Petrologia magnética do granito anorogênico Bannach, terreno granito greenstone de Rio Maria, Pará. Revista Brasileira de Geociências, 37:17-36

Althoff F.., Barbey P., Boullier A.M. 2000. 2.8-3.0 Ga plutonism and deformation in the SE Amazonian craton: the Archean granitoids of Marajoara, Carajás Mineral province; Brazil. Precambrian Research, 104:187-206. https://doi.org/10.1016/S0301-9268(00)00103-0

Anderson J.L. 1980. Mineral equilibria and crystallization conditions in the late Precambrian Wolf River rapakivi massif. American Journal of Science, 280:289-332. DOI: 10.2475/ajs.280.4.289

Anderson J.L., Barth A.P., Wooden J.L., Mazdab F. 2008. Thermometers and thermobarometers in granitic systems. Reviews in Mineralogy and Geochemistry, 69:121-142. https://doi.org/10.2138/ rmg.2008.69.4

AndersonJ.L.,BenderE.E.1989.Natureandorigin of Proterozoic A-Type granitic magmatism in the southwestern United States of America. Lithos, 23:19-52. https://doi.org/10.1016/0024-4937(89)90021-2

Anderson J.L., Morrison J. 2005. Ilmenite; magnetite; and peraluminous Mesoproterozoic anorogenic granites of Laurentia and Baltica. Lithos, 80:45-60. https://doi.org/10.1016/j. lithos.2004.05.008

Anderson J.L., Smith D.R. 1995. The Effects of temperature and $\mathrm{fO}_{2}$ on the Al-in-hornblende barometer. American Mineralogist, 80:549559. DOI: $10.2138 / \mathrm{am}-1995-5-614$

Barbosa A.A., Lafon J.M., Neves A.P., Vale A.G. 1995. Geocronologia $\mathrm{Rb}-\mathrm{Sr}$ e $\mathrm{Pb}-\mathrm{Pb}$ do Granito Redenção; SE do Pará: Implicações para a evolução do magmatismo Proterozóico da região de Redenção. Boletim do Museu Paraense Emílio Goeldi; série Ciências da Terra, 7:147-164

Blundy J.D., Holland T.J.B. 1990. Calcic amphibole equilibria and a new amphibole plagioclase geothermometer. Contributions Mineralogy Petrology, 104:208-224. https://doi.org/10.1007/ BF00306444

Boehnke P., Watson E.B., Trail D., Harrison T.M., Schmitt A.K. 2013. Zircon saturation re-revisited. Chemical Geology, 351:324-334. https://doi.org/10.1016/j.chemgeo.2013.05.028

Buddington A.F., Lindsley D.H. 1964. Iron-titanium oxide minerals and synthetic equivalents. Journal of Petrology, 5(2):310-357. DOI: 10.1093/petrology/5.2.310
Carmichael IS.E 1966. The iron-titanium oxides of salic volcanic rocks and their associated ferromagnesian silicates. Contributions to Mineralogy and Petrology, 14(1):36-64. https://doi.org/10.1007/ BF00370985

Carmichael I.S.E. 1991. The redox states of basic and silicic magmas: a reflection of their source regions. Contributions to Mineralogy and Petrology, 106:129-141. https://doi.org/10.1007/BF00306429

Carvalho T.A. 2017. Petrografia, Geoquímica e Suscetibilidade Magnética da Porção Leste do Granito Gradaús, Província Carajás, SE do Pará. Master Dissertation, Universidade Federal do Pará, Belém, $61 \mathrm{p}$

Clark D.A. 1999. Magnetic Petrology of igneous intrusion: implications for exploration and magnetic interpretation. Exploration Geophysics, 30:5-26. DOI: 10.1071/EG999005

Cunha I.R.V., Dall'Agnol R., Feio G.R.L. 2016. Mineral Chemistry and Magnetic Petrology of the Archean Planalto Suíte, Carajás Province - Amazonian Craton: Implications for the Evolution of Ferroan Archean Granites. Journal of South American Earth Sciences, 67:100-121. DOI: 10.1016/j.jsames.2016.01.007

Dall'Agnol R. 1982. Maçiço Jamon: Evolução Petrológica de um granito da Amazônia Oriental. In: Simpósio de Geologia da Amazônia, 1, 1982, Belém. Anais..., 2:139-161.

Dall'Agnol R., Costi H.T., Leite A.A., Magalhães M.S., Teixeira N.P. 1999a. Rapakivi granites from Brazil and adjacent areas. Precambrian Research, 95:9-39. DOI: 10.1016/S0301-9268(98)00125-9

Dall'Agnol R., Cunha I.R.V., Guimarães F.V., Oliveira D.C., Teixeira M.F.B., Feio G.R.L., Lamarão C.N. 2017. Mineralogy, geochemistry, and petrology of Neoarchean ferroan to magnesian granites of Carajás Province, Amazonian Craton: The origin of hydrated granites associated with charnockites. Lithos, 277:3-32. DOI: 10.1016/j. lithos.2016.09.032

Dall'Agnol R., Oliveira D.C. 2007. Oxidized, magnetite series, rapakivitype granites of Carajás, Brazil: implications for classification and petrogenesis of A-type granites. Lithos, 93(3-4):215-233. https://doi. org/10.1016/j.lithos.2006.03.065

Dall'Agnol R., Oliveira D.C., Guimarães F.V., Gabriel E.O., Feio G.R.L., Lamarão C.N., Althoff F.J., Santos P.A., Teixeira M.F.B., Silva A.C., Rodrigues D.S., Santos M.J.P., Silva C.R.P, Santos R.D., Santos P.J.L. 2013. Geologia do Subdomínio de Transição do Domínio Carajás - Implicações para a evolução arqueana da Província Carajás - Pará. In: Simpósio de Geologia da Amazônia, 13., Belém. Anais... SBG. CD-ROM

Dall'Agnol R., Pichavant M., Champenois M. 1997. Iron-Titanium Oxide Minerals of the Jamon Granite; Eastern Amazonian Region; Brazil: Implications for the Oxigen Fugacity in Proterozoic, A-type Granites. Anais da Academia Brasileira de Ciências, 69(3):324-347.

Dall'Agnol R., Rämö O.T., Magalhães M.S., Macambira M.J.B. 1999b. Petrology of the anorogenic, oxidised Jamon and Musa granites; Amazonian craton: implications for the genesis of Proterozoic A-type granites. Lithos, 46:431-462. DOI: 10.1016/S0024-4937(98)00077-2

Dall'Agnol R., Scaillet B., Pichavant M. 1999c. An experimental study of the Lower Proterozoic A-type Granite from the Eastern Amazonian craton, Brazil. Journal of Petrology, 40(11):1673-1698. https://doi.org/10.1093/petroj/40.11.1673

Dall'Agnol R., Teixeira N.P., Rämö O.T., Moura C.A.V., Macambira M.J.B., Oliveira D.C. 2005. Petrogenesis of the Paleoproterozoic, rapakivi, A-type granites of the Archean Carajás Metallogenic Province, Brazil. Lithos, 80:101-129. https://doi.org/10.1016/j. lithos.2004.03.058 
Dall'Agnol R., Oliveira M.A., Almeida J.A.C., Althoff F.J., Leite A.A.S., Oliveira D.C., Barros C.E.M. 2006. Archean and Paleoproterozoic granitoids of the Carajás metallogenic province, eastern Amazonian craton. In: Dall'Agnol R., Rosa-Costa L.T., Klein E.L. (eds.). Symposium on Magmatism, Crustal Evolution, and Metallogenesis of the Amazonian Craton. Abstracts Volume and Field Trips Guide. Belém, PRONEX-UFPA/SBG-NO, 150p.

Deer W.A., Howie R.A., Zussman J. 1992. Rock-forming Minerals. $2^{\text {a }}$ ed. London, Longmans, 696 p.

Duarte K.D. 1992. Geologia e geoquímica do Granito Mata Surrão (SW de Rio Maria-PA): um exemplo de granito "stricto sensu" Arqueano. Dissertation, Universidade Federal do Pará, Centro de Geociências, Belém, 217 p.

Elliott B.A. 2001. Crystallization conditions of the Wiborg rapakivi batholith, SE Finland: an evaluation of amphibole and biotite mineral chemistry. Mineralogy Petrology, 72:305-024. https://doi. org/10.1007/s007100170021

Erdmann S., Martel C., Pichavant M., Kushnir A. 2014. Amphibole as an archivist of magmatic crystallization conditions: problems, potential, and implications for inferring magma storage prior to the paroxysmal 2010 eruption of Mount Merapi, Indonesia. Contributions to Mineralogy and Petrology, 167:1-23. https://doi. org/10.1007/s00410-014-1016-4

Frost B.R. 1991. Introduction to oxygen fugacity and its petrologic importance. In: Lindsley D.H. (Ed.). Oxide Minerals: Petrologic and Magnetic Significance. Mineralogical Society of America. Reviews in Mineralogy, 25:1-9.

Frost B.R., Lindsley D.H. 1991. Ocurrence of iron-titanium oxides in igneous rocks. In: Lindsley D.H. (Ed.). Oxide Minerals: Petrologic and Magnetic Significance. Mineralogical Society of America. Reviews in Mineralogy, 25:433-468.

Frost C.D., Frost B.R., Chamberlain K.R., Edwards B. 1999. Petrogenesis of the 1.43 Ga Sherman Batholith, SE Wyoming, USA: a reduced, rapakivi-type anorogenic granite. Journal of Petrology, 40:1771-1802. DOI: 10.1093/petrology/40.12.1771

Frost B.R., Frost C.D., Hulsebosch T.P., Swapp S.M. 2000. Origin of the charnockites of the Louis Lake batholith, Wind river range, Wyoming. Journal of Petrology, 40:1771-1802.

Gastal M.C.P. 1987. Petrologia do Maçiço Granítico Musa, Sudeste do Pará. Dissertation, Centro de Geociências, Universidade Federal do Pará, Belém, 316 p.

Haggerty S.E. 1991. Oxide textures a mini-atlas. In: Lindsley D.H. (Ed.). Oxide Minerals: Petrologic and Magnetic Significance. Mineralogical Society of America. Reviews in Mineralogy, 25:129-219.

Hammarstron J.M., Zen E.A. 1986. Aluminium in hornblende: an empirical igneous geobarometer. American Mineralogist, 71:1297-1313.

Hawthorne F.C., Oberti R., Harlow G.E., Maresch W.V., Martin, R.F., Schumacher J.C., Welch, M.D. 2012. Nomenclature of the amphibole supergroup. American Mineralogist, 97:2031-2048. DOI: 10.2138/ am.2012.4276

Hollister L.S., Grisson G.C., Peters E.K., Stowell H.H., Sisson V.B. 1987. Confirmation of the empirical correlation of $\mathrm{Al}$ in hornblende with pressure of solidification of calc-alkaline plutons. American Mineralogist, 72:231-239.

Huhn S.R.B., Santos A.B.S., Amaral A.F., Ledsham E.J., Gouveia J.L., Martins L.B.P., Montalvão R.M.G., Costa V.G. 1988. O terreno granitogreenstone da região de Rio Maria - Sul do Pará. In: Congresso Brasileiro de Geologia, 35., Belém. Anais... 3:1438-1453.

Ishihara S. 1981. The granitoid series and mineralization. In: Skinner B.J. (Ed.). Economic Geology, 75th anniversary volume, 458-484.
Johnson M.C., Rutherford M.J. 1989. Experimental calibration of the aluminium-in hornblende geobarometer with application to Long Valley caldera (California) volcanic rocks. Geology, 17:837841. https://doi.org/10.1130/0091-7613(1989)017\%3C0837:ECO TAI\%3E2.3.CO;2

Klimm K., Holtz F., Johannes W., King P.L. 2003. Fractionation of metaluminous A-type granites: an experimental study of the Wangrah Suite, Lachlan Fold Belt, Australia. Precambrian Research, 124:327-341. DOI: 10.1016/S0301-9268(03)00092-5

Kosunen P.J. 2004. Petrogenesis of Mid-Proterozoic A-type Granite: Case Studies from Fennoscandia (Finland) and Laurentia (New Mexico). Thesis, Department of Geology, University of Helsinki.

Kowallis B.J., Christiansen E.H., Griffen D.T. 1997. Compositional variations in titanite. Geological Society of American Abstracts with Programs, 29(6):402.

Lamarão C.N., Dall'Agnol R. 2004. Química mineral de anfibólios e biotitas e condições de cristalização de granitoides paleoproterozoicos da região de Vila Riozinho, Província Aurífera do Tapajós, Cráton Amazônico. Revista Brasileira de Geociências, 34:95-108.

Leake B.E., Wooley A.R., Arps C.E.S., Birch W.D., Gilbert M.C., Grice J.D., Hawthorne F.C., Kato A., Kisch H.J., Krivovichev V.G., Linthout K., Laird J., Mandarino J.A., Maresch W.V., Nickel E.H., Rock N.M.S., Schumacher J., Smith J.C., Stephenson N.C.N. Whittaker E.J.W., Youzhi G. 1997. Nomenclature of amphiboles: report of the subcommittee on amphiboles of the international mineralogical association commission on new minerals and Mineral names. Mineralogical Magazine, 61:295-321.

Leite A.A.S. 2001. Geoquímica, petrogênese e evolução estrutural dos granitóides arqueanos da região de Xinguara, SE do Cráton Amazônico. Thesis, Pós-Graduação em Geoquímica e Petrologia, Centro de Geociências, Universidade Federal do Pará, Belém, 330 p.

Le Maitre R.W., Streckeisen A., Zanettin B., Le Bas M.J., Bonin B., Bateman P., Bellieni G., Dudek A., Efremova J., Keller J., Lameyre J., Sabine P.A., Schmidt R., Sørensen H., Woolley A.R. 2002. Igneous Rocks. A Classification and Glossary of Terms. Recommendations of the International Union of Geological Sciences Subcommission on the Systematics of Igneous Rocks. Cambridge, Cambridge University Press, $236 \mathrm{p}$.

Lima P.H.A., Lamarão C.N., Santos M.J.P. 2014. Petrografia, geoquímica e suscetibilidade magnética do Granito Paleoproterozoico São João, sudeste do Cráton Amazônico, Província Carajás. Boletim do Museu Paraense Emílio Goeldi, Ciências Naturais, 9:47-72.

Macambira M.J.B., Lafon J.M. 1995. Geocronologia da Província Mineral de Carajás, Síntese dos dados e novos desafios. Boletim do Museu Paraense Emílio Goeldi, Ciências Naturais, 7:263-287.

Machado N., Lindenmayer Z., Krogh, T.E., Lindenmayer D. 1991. U-Pb geochronology of Archean magmatism and basement reactivation in the Carajás Área, Amazon Shield, Brazil. Precambrian Research, 49:329-354. https://doi.org/10.1016/0301-9268(91)90040-H

Miller C.F., McDowell S.M., Mapes R.W. 2003. Hot and cold granites? Implications of zircon saturation temperatures and preservation of inheritance. Geology, 31:529-532. https://doi. org/10.1130/0091-7613(2003)031\%3C0529:HACGIO\%3E2.0.CO;2

Montalvão R.M.G., Bezerra P.E.L., Prado P., Fernandes C.A.C., Silva G.H., Brim R.J.P. 1982. Características petrográficas e geoquímicas do Granito Redenção e suas possibilidades metalogenéticas. In: Congresso Brasileiro de Geologia, 32., Salvador. Anais... 2:520-548.

Mutch E.J.F., Blundy J.D., Tattitch B.C., Cooper F.J., Brooker R.A. 2016. An experimental study of amphibole stability in low-pressure granitic magmas and a revised Al-in-hornblende geobarometer. Contributions to Mineralogy and Petrology, 171:85. https://doi. org/10.1007/s00410-016-1298-9 
Nachit H. 1994. Contribution à la typologie des granitoides, Petrogenèse et pétrologie structurale du batholith panafricain $d u$ cercle de Tafroute boutonniére de Kerdous, Anti-Atlas occidental. Maroc). Thesis, Université Ibhou Zohor, Agadir, 465 p.

Nachit H., Razafimahefa N., Stussi J.M., Carron J.P. 1985. Composition chimique des biotites et typologie magmatique des granitoïdes. Comptes Rendus de l'Académie des Sciences, 301:813-818.

Oliveira D.C., Dall'Agnol R., Barros C.E.M., Oliveira M.A. 2009. Geology, geochemistry and magmatic evolution of the Paleoproterozoic, anorogenic oxidized A-type Redenção granite of the Jamon Suíte, eastern Amazon Craton, Brazil. Canadian Mineralogist, 47(6):14411468. DOI: 10.3749/canmin.47.6.1441

Oliveira D.C., Dall'Agnol R., Silva J.B.C., Almeida J.A.C. 2008. Gravimetric, radiometric, and magnetic susceptibility study of the Paleoproterozoic Redenção and Bannach plutons: implications for architecture and zoning of A-type granites. Journal of South American Earth Sciences, 25:100-115. DOI: 10.1016/j.jsames.2007.10.003

Oliveira D.C., Neves S.P., Trindade R.L.F., Dall'Agnol R., Mariano G., Correia P.B. 2010a. Magnetic anisotropy of the Redenção granite, eastern Amazonian craton (Brazil): Implications for the emplacement of A-type plutons. Tectonophysics, 493:27-41. http:// dx.doi.org/10.1016/j.tecto.2010.07.018

Oliveira M.A., Dall'Agnol R., Althoff F.J., Leite A.A.S. 2009b. Mesoarchean sanukitoid rocks of the Rio Maria Granite-greenstone Terrane, Amazonian craton, Brazil. Journal of South American Earth Sciences, 27:146-160. https://doi.org/10.1016/j.jsames.2008.07.003

Oliveira M.A., Dall'Agnol R., Scaillet B. 2010c. Petrological constraints on crystallization conditions of Mesoarchean Sanukitoid Rocks, southeastern Amazonian Craton, Brazil. Journal of Petrology, 51:2121-2148. https://doi.org/10.1093/petrology/egq051

Paiva Jr. A.L., Lamarão C.N., Lima P.H.A. 2011. Geologia, Petrografia e Geoquímica do Batólito Seringa, Província Carajás, SSE do Pará. Revista Brasileira de Geociências, 41(2):185-202.

Putirka K. 2016. Amphibole thermometers and barometers for igneous systems and some implications for eruption mechanisms of felsic magmas at arc volcanoes. American Mineralogist, 101(4):841858. https://doi.org/10.2138/am-2016-5506

Rämö O.T., Haapala I. 1995. One hundred years of rapakivi granite. Mineralogy and Petrology, 52:129-185. https://doi.org/10.1007/ BF01163243
Ridolf F., Renzulli A. 2012. Calcic amphiboles in calc-alkaline and alcaline magmas: thermobarometric and chemometric empirical equations valid up to $1130^{\circ} \mathrm{C}$ and $2.2 \mathrm{GPa}$. Contributions to Mineralogy and Petrology, 163:877-895. https://doi.org/10.1007/ s00410-011-0704-6

Ridolfi F., Renzulli A., Puerini M. 2010. Stability and chemical equilibrium of amphibole in calc-alkaline magmas: an overview, new thermobarometric formulations, and application to subduction-related volcanoes. Contributions to Mineralogy and Petrology, 160:45-66. https://doi.org/10.1007/ s00410-009-0465-7

Rocha Jr. G.L.D. 2004. Caracterização petrográfica do Granito Paleoproterozóico Marajoara, Terreno Granito-Greenstone de Rio Maria, SE do estado do Pará. Course Conclusion Paper, Centro de Geociências, Universidade Federal do Pará, Belém. 45p.

Schmidt M.W. 1992. Amphibole composition in tonalite as a function of pressure: an experimental calibration of the Al-in-hornblende barometer. Contributions to Mineralogy and Petrology, 110:304-310. https://doi.org/10.1007/BF00310745

Schumacher J.C. 1997. The estimation of ferric iron in electron micropobe analysis of amphiboles. In: Leake B.E. (Ed.) Nomenclature of Amphiboles. Reportof the Subcommittee on Amphiboles of the International Mineralogical Association Commission on New Minerals and Mineral Names. European Journal of Mineralogy, 9:623-651

Vasquez L.V., Rosa-Costa L.R., Silva C.G., Ricci P.F., Barbosa J.O., Klein E.L., Lopes E.S., Macambira E.B., Chaves C.L., Carvalho J.M., Oliveira J.G., Anjos G.C., Silva H.R. 2008. Geologia e Recursos Minerais do Estado do Pará: Sistema de Informações Geográficas - SIG: texto explicativo dos mapas Geológicos e Tectônico e de Recursos Minerais do Estado do Pará, 328p.

Vigneresse J.L. 2005. The specific case of the Mid-Proterozoic rapakivi granites and associated suite within the context of Columbia supercontinent. Precambrian Research, 137:1-34. DOI: 10.1016/j.precamres.2005.01.001

Watson E.B., Harrison T.M. 1983. Zircon saturation revisited temperature and composition effects in a variety of crustal magma types. Earth and Planetary Science Letters, 64:295-304. https://doi. org/10.1016/0012-821X(83)90211-X

Wones D.R. 1989. Significance of the assemblage titanite + magnetite + quartz in granitic rocks. American Mineralogist, 74:744-749. 